\title{
Molecular functionalization of 2D materials
}

\author{
Miriam C. Rodríguez González, a* Rahul Sasikumara and Steven De Feyter ${ }^{a *}$ \\ aDepartment of Chemistry, Division of Molecular Imaging and Photonics, KU Leuven, Celestijnenlaan 200F, \\ B-3001 Leuven, Belgium
}

In this chapter we give an overview of different chemical modifications that can be done on the surface of two-dimensional (2D) layered materials. We place emphasis on the diversity of reactions that have been proposed and are now available to surface scientists working in 2D materials field. Using mainly, but not exclusively, MoS2 as example, reactions involving covalent and non-covalent interactions are discussed.

\section{INTRODUCTION}

With the isolation of graphene in 2004, a material theoretically predicted much earlier, materials chemistry has been dominated by two-dimensional (2D) materials in the $21^{\text {st }}$ century. ${ }^{1,2} \mathrm{~A} 2 \mathrm{D}$ material can be seen as a monolayer-thick inorganic crystal extending only in two dimensions. In the last decade, we have witnessed the emergence of new 2D layered materials from previously known 3D solid state structures based on stacked 2D layers. Some examples of these materials are the transition-metal dichalcogenides $(T M D C s),{ }^{3}$ such as molybdenum disulfide $\left(\mathrm{MoS}_{2}\right)$; hexagonal boron nitride (h-BN); ${ }^{4}$ black phosphorus (BP) ${ }^{5}$ or germanene. ${ }^{6} \mathrm{~A}$ wide-ranging set of properties become accessible through these different materials. For example, in terms of electronic properties, $\mathrm{h}-\mathrm{BN}$ is an insulator while TMDCs such as $\mathrm{MoS}_{2}$ and $\mathrm{NbSe}_{2}$ exhibit different behavior, being direct band gap semiconductor and metallic, respectively. After the success in the isolation and processing of these $2 \mathrm{D}$ materials, the attention has been focused on the characterization of their special properties and the evaluation of its applicability.

Due to their high surface/volume ratio, 2D materials are sensitive to surface modification via chemical functionalization. Experimentally, different molecular chemistry approaches based on covalent or noncovalent interactions between pristine or defective TMDCs and organic moieties have been proposed. The simplest way for the functionalization is the physisorption of molecules via solution or gas phase in a nonordered fashion. An additional level of control can be reached though via the formation of ordered supramolecular assemblies. Non-covalent molecular functionalization of materials concerns with properties that emerge from electrostatic and dipole-dipole or induced dipole interactions, $\mathrm{H}$-bonds, or van der Waals interactions. These non-covalent approaches allow for surface modification in a straightforward manner and they do not require specific molecule-substrate bonds. Despite the feasibility of the non-covalent protocols, in the last years, the interest in covalent functionalization approaches has increased. These covalent methodologies use specific chemical interactions with the substrate which can lead to the formation of strong and stable covalent bonds, with changes in the electronic structure of the material as a consequence. For example, the modification of the band gap on monolayered $\mathrm{MoS}_{2}$ materials 
can be achieved through these covalent interactions ${ }^{7}$, although some examples based on non-covalent interactions of strong organic dopants for this purpose can be found as well. ${ }^{8}$ It has been theoretically predicted that the donor/acceptor nature of the substituents of the molecule and the nature of the bond on the surface can be systematically used to tune the band structure of the material on demand. ${ }^{9}$ At first glance, covalent functionalization would seem to be superior to non-covalent methods due to the strength and stability of the interactions involved. But the large surface-to-volume ratios in 2D materials means that their properties are highly sensitive to external conditions, and covalent bonds could irreversibly deteriorate the interesting properties of the pristine material. For instance, strong chemical bonds have been shown to induce phase change from semiconducting $2 \mathrm{H}-\mathrm{MoS}_{2}$ into metallic $1 \mathrm{~T}-\mathrm{MoS}_{2} \cdot{ }^{10}$ For these reasons, before proceeding to the chemical functionalization of a material, an evaluation of the properties required in the processed material and the advantages/drawbacks of the protocol chosen has to be carried out.

The importance of surface modification lies in the ease and selectivity to control the surface composition, structure, and properties of the material. 2D nanosheets of TMDCs have been extensively used in the last decade in multiple applications such as catalysis, energy storage, nanoelectronics, photonics or sensing. ${ }^{11}$ With the chemical functionalization at the molecular level it is possible to tune or engineer the electrical, optical, or thermal properties exhibited by these materials in a rational fashion, leading to promising opportunities for applications. Some of the advantages include the formation of hybrid structures or improved dispersibility ${ }^{12}$ of the material for further processing.

In this work, we focus on the advances made in the functionalization of transition metals dichalcogenides (TMDCs), with special attention to the most intensively studied TMDC, MoS2. Single sheets of TMDCs consist of an atomic layer of transition metals ( $M=M o, W$, Ti, etc.) between two atomic layers of chalcogens $(X=S$, Se or Te) in a sandwich-type structure. TMDCs exist in several structural phases depending on the stacking of the atomic planes of the material. Commonly, we find structural phases with a trigonal prismatic $(2 \mathrm{H})$ or octahedral $(1 \mathrm{~T})$ coordination of metal atoms. ${ }^{13}$ For MoS2, several polymorphs can be found, the most important ones being the metallic 1T phase (1T-MoS $)$ and the semiconducting $2 \mathrm{H}$ $\left(2 \mathrm{H}-\mathrm{MoS}_{2}\right)$ and $3 \mathrm{R}\left(3 \mathrm{R}-\mathrm{MoS}_{2}\right)$ phases. (Figure 1) Although the $2 \mathrm{H}-\mathrm{MoS}_{2}$ phase is thermodynamically stable, the chemical exfoliation of bulk $\mathrm{MoS}_{2}$ by lithium intercalation (ceMoS $)$ induces a phase transition from $2 \mathrm{H}$ $\mathrm{MoS}_{2}$ to the metallic $1 \mathrm{~T} / 1 \mathrm{~T}^{\prime}$ polytype ( $\approx 65 \%$ of transformation). ${ }^{14}$ 


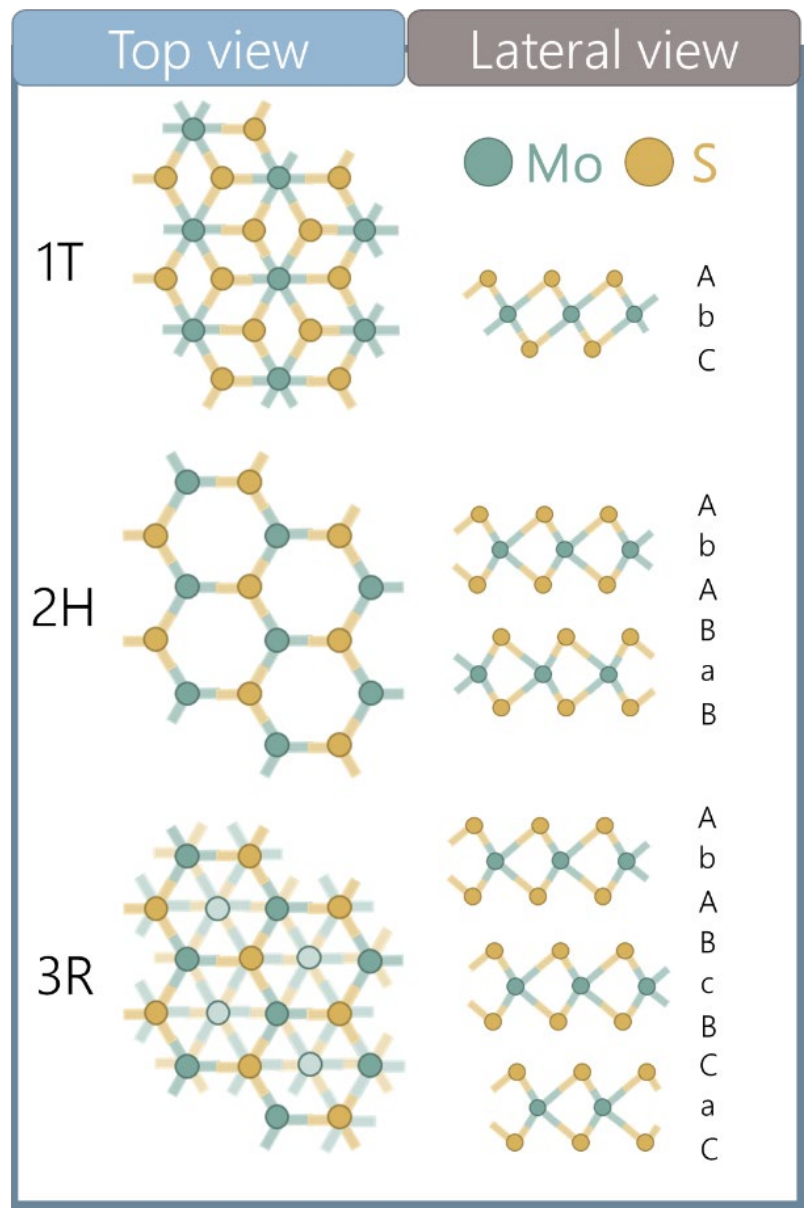

Figure 1. Scheme for the crystal structure of the different polytypes of MoS2 (1T, $2 \mathrm{H}$ and $3 \mathrm{R})$. Adapted with permission from reference 11. Copyright 2015 The Royal Chemical Society.

In terms of reactivity, the semiconducting phase $\left(2 \mathrm{H}-\mathrm{MoS}_{2}\right)$ cannot be easily functionalized, requiring in most of the cases the incorporation of defects or vacancies for its functionalization. In contrast, in the metallic phases (1T-MoS 2 or its distorted structures $\left.1 T^{\prime}-M_{0} S_{2}\right)$ strong bonds can be formed between molecules (especially electrophiles) and the surface due to the possibility of a direct molecule-substrate electron transfer. As commented before, these atomic-thick materials have interesting properties such as a direct bandgap or a strong spin-orbit coupling.

Here, we describe the experimental approaches for the molecular modification of 2D TMDCs that have been explored so far, in particular those applied for the model system of these materials, MoS 2 . However, these modification strategies can also be applied to other TMDCs such as WS2 or MoSe2.

\section{NON-COVALENT FUNCTIONALIZATION OF 2D MATERIALS}

Non-covalent functionalization is desirable as it is a non-destructive and reversible process. Weak interactions do not necessarily mean that the resulting property modulations are insignificant. For instance, for a molecule adsorbed on a 2D material, when the energy of the highest occupied molecular orbital (HOMO) of the molecule is higher than the Fermi level of the material, charge transfer pathways where 
electron density is transferred between the molecules and the material can be opened. Such transfer of electron density can influence electrical properties in ultrathin materials. This effect is known as contact doping or charge transfer doping. ${ }^{15}$

The picture of a monolayer of few molecules influencing the properties of a material might come off as surprising. It is compelling to ask how such a minor influence of molecules can be as effective on ultrathin materials, as conventional doping is on traditional semiconductors. The answer lies in the size of the materials in our discussion. 2D materials are characteristically different from conventional materials as they lack a bulk. This low dimensionality leads, as pointed out before, to extremely high surface-to-volume ratios in these materials.

Apart from these, many of their optical properties are strongly influenced by external factors as their monolayer nature stabilizes various many-body exciton effects. ${ }^{16}$ The extreme sensitivity of these materials is a dream come true for every researcher. Unfortunately, high sensitivity without selectivity is a doubleedged sword. Due to the extent to which immediate surroundings of 2D materials influence their properties, significant predictable results are only obtained with a good control of the environment. For example, adsorbed water molecules at the surface can lead to charge trapping states which induce a hysteresis in current response of $\mathrm{MoS}_{2}$ field-effect transistor (FET) devices. ${ }^{17}$ The photosensitivity of MoS2 multiplies this hysteresis on shining light, as more charges are generated and populate the trap states. Some reports claim that adsorption of water quenches the photoluminescence (PL) in single layer (SL$\left.\operatorname{MoS}_{2}\right){ }_{1}^{18}$ although this effect remains under discussion. These results are an outcome of the polar nature of water. Water molecules adsorb at the interface orienting to form a template adlayer. This adlayer acts as an n-type dopant that quenches radiative recombination in $\mathrm{MoS}_{2}$. Recent studies have shown that common organic solvents also have a similar influence on the electronic and optoelectronic properties of TMDCs. Samori and co-workers report doping in monolayer $\mathrm{MoS}_{2}$ and $\mathrm{WSe}_{2}$ by the controlled physisorption of benzene derivatives. They observe that the intensity and direction of doping depend not only on the functional group on the adsorbed molecule but also on the thermodynamic stability of the physisorption. ${ }^{19}$ In another study, Tian and co-workers report that several common solvents could influence the electrical properties of TMDCs. ${ }^{20}$ The doping effect is again influenced by different contributions: the charge donating characteristics and dipole interactions of the molecule, and the surface energy of the solvent. The effect of solvents could be eliminated from the surface by common washing procedures in most cases. But one particular solvent, N-methyl-2-pyrrolidone (NMP), could not be completely removed from the surface.

Beyond exploitable results, these experiments are more examples of the sensitivity of 2D materials than potential applications. These works succeed in showcasing the promises and pitfalls in the field of 2D materials functionalization. Any influence on electrical and optical properties of a 2D material must be viewed with scrutiny. Utmost caution and a good control of the environment is essential due to the extreme sensitivity of the systems in hand.

Our discussion on non-covalent functionalization of 2D materials is divided into two sections; a) gas phase functionalization, and b) condensed-phase functionalization. 


\subsection{GAS PHASE FUNCTIONALIZATION}

Non-covalent gas-phase functionalization protocols are among the least explored areas of functionalizing 2D materials. Quite some literature predicts interesting properties of gas adsorbed 2D materials. Experimental results have remained relatively scarce, some of which will be discussed here. One of the earlier reports of gas phase non-covalent functionalization of TMDCs is by Wu and co-workers. They reported an enhancement in the PL of annealed TMDCs materials through a charge transfer to physisorbed $\mathrm{O}_{2}$ and $\mathrm{H}_{2} \mathrm{O}$ molecules. ${ }^{16} \mathrm{PL}$ intensity of post-annealed $\mathrm{MoS}_{2}$ is enhanced 10 times in the presence of $\mathrm{H}_{2} \mathrm{O}$, 35 times in the presence of $\mathrm{O}_{2}$, and 100 times when they are present together. PL intensities reverted as the gases were removed, indicating that the change in the PL intensity is related to the non-covalent interactions of the gas molecules with the surface. These responses were also used to produce highly sensitive humidity sensors in the range of 0-35\% humidity, beyond which the PL response saturates. ${ }^{21}$ Monolayers of MoS 2 prepared by chemical vapor deposition (CVD) over oxide materials are unintentionally $\mathrm{n}$-doped and have an excess of electrons. These excess electrons promote the formation of negative trions over excitons on irradiation. These decrease the PL intensity due to a competition between radiative and non-radiative recombination pathways in trions. With the annealing of the samples, a depletion of the excess electrons and improved PL of the material can be achieved. In the above study, MoS 2 samples annealed at $450^{\circ} \mathrm{C}$ for 40 minutes displayed the strongest PL enhancement among samples annealed for shorter and longer times. Lee and co-workers reported the work function modulation of $\mathrm{MoS}_{2}$ by $0.4 \mathrm{eV}$ (4.04-4.47 eV) through an oxygen-adsorption assisted pseudo p-n junction. ${ }^{22}$ This was achieved from a relatively small surface coverage of oxygen molecules $(<10 \%)$. Their theoretical model suggests that the work function could be modulated up to a range of $1.2 \mathrm{eV}$ by varying the surface oxygen coverage between $0-100 \%$.

Another relevant work is from the group of Eom and co-workers where they report the functionalization of single, bi, and few layer MoS 2 devices with $N_{2}$ in presence UV light (Figure 2). ${ }^{23} N_{2}$ doping shifts the threshold voltage of $\mathrm{MoS}_{2}$ FETs towards negative gate voltages. It also improves the carrier density and charge-carrier mobility of n-type MoS2 non-destructively. The prior electrical characteristics are completely recovered by treating the samples with $\mathrm{O}_{2}$ under deep ultraviolet (DUV) light. First-principles calculations including van der Waals interactions have been carried out to predict adsorption energies of different gases. ${ }^{24}$ The adsorption energy is different for each molecule, being in the range of -0.07 to $-0.15 \mathrm{eV}$ for $\mathrm{N}_{2}$ depending on the functional used in the calculations. Therefore, the improved properties are a result of the physisorption of $\mathrm{N}_{2}$ at the interface. The doping is remarkably stable in ambient air and a non-significant loss of doping was reported even after 20 days of exposure. 

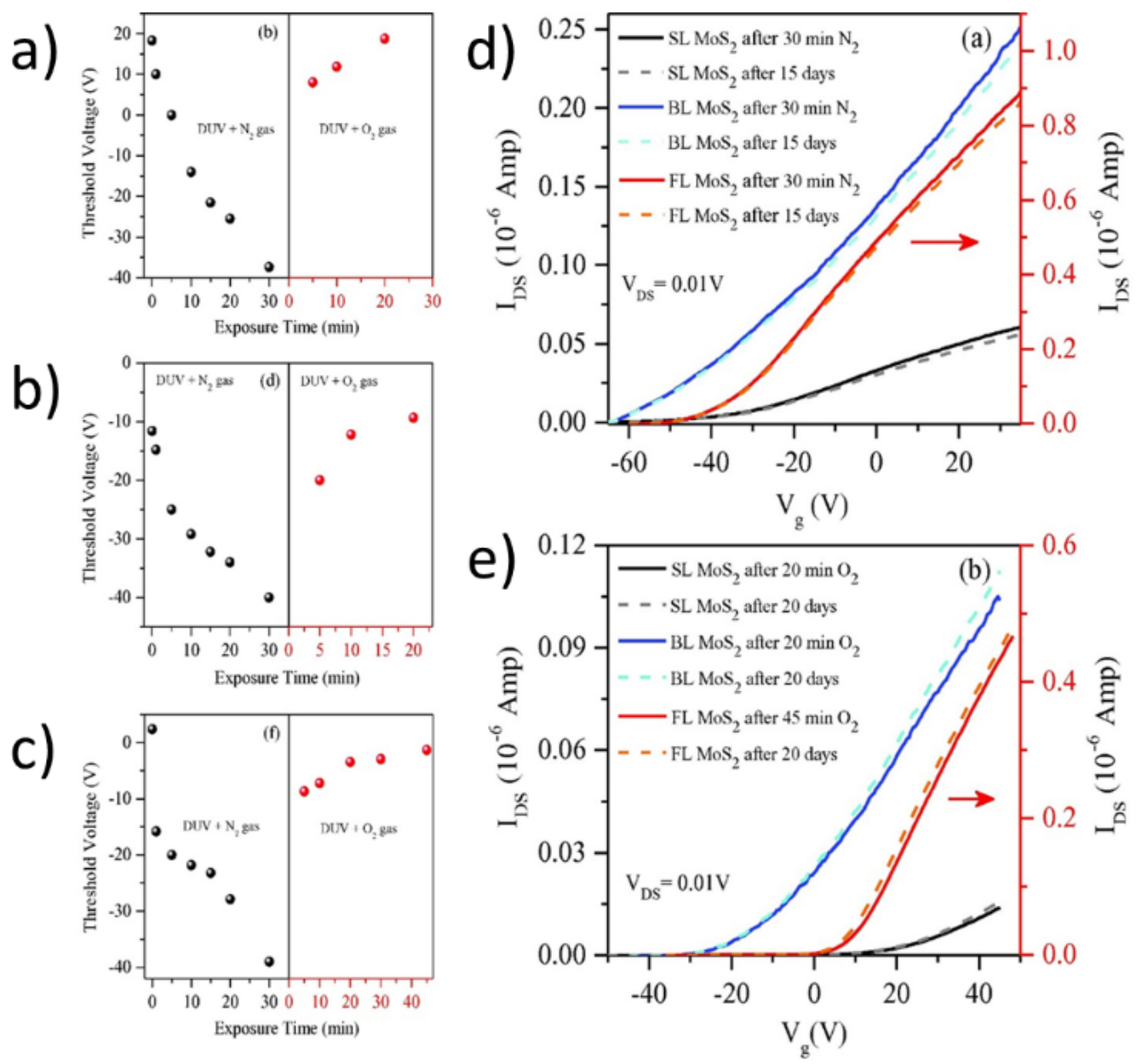

Figure 2. Dependence of the threshold voltage on time of exposure for $\mathrm{N}_{2}$ and $\mathrm{O}_{2}$ in presence of DUV light for a) single, b) bi and c) few layer $\mathrm{MoS}_{2}$. d) Stability of $\mathrm{N}_{2}$ doping of SL (black), BL (blue) and FL (red) MoS transistors as revealed by a I(DS)- $V_{g}$ plot after exposure to $N_{2}$ molecules and DUV light, and the same plot after 15 days. e) same stability test but after recovery of the $\mathrm{MoS}_{2}$ transistor with $\mathrm{O}_{2}$ molecules and DUV light, and the same plot after 20 days. Reprinted with permission from reference 21. Copyright 2014 Wiley-VCH.

Non-covalent functionalization in gas-phase has not attracted the same vigour of interest when compared to the condensed phase approaches. There is only limited room for tuning the properties of a molecule before it condenses to form a liquid or a solid. Hence, gas-phase strategy falls short in terms of degree of tunability when compared to the condensed phase techniques. This, in combination with the requirement of working in high vacuum deters the commercialization of these functionalization processes. Consequently, even if UHV conditions offer the better mechanistic understanding of the surface interactions, UHV is not suitable for most of the real-life applications of these materials.

\subsection{CONDENSED PHASE FUNCTIONALIZATION}

We have seen above the drawbacks of gas phase functionalization. Condensed phase reactions generally do not require an extreme control over external conditions. Additionally, there is a higher ceiling on tunability compared to gas phase techniques as there is an additional control in the form of solvent 
medium. The ease of performing reactions in liquid phase makes them instantly attractive. Our discussion here will focus on condensed phase techniques that are based on self-assembly of the interacting molecules. We will have a brief discussion on functionalization methods which are not based on selfassembly in the latter part of is section.

\subsubsection{CONDENSED PHASE TECHNIQUES BASED ON SELF-ASSEMBLY}

The impact of an individual small molecule on the properties of a surface is in most of the cases not significant and/or detectable. However, a stable array of several packed and oriented molecules can be used to successfully tune the properties and behavior of these materials. Self-assembly is an association of molecules through weak and reversible interactions to form organized polymolecular systems. Such an ordered association allows a magnification of molecular properties by directional orientation and hence greater contact potential. Such control also leads to an improved understanding of the interface. Combined with the functional diversity of organic molecules, such self-assembled systems are ideal for tuning the properties of 2D materials. One should differentiate two self-assembly approaches. The first one is based on the formation of densely packed self-assembled monolayers (SAMs) where the molecules form a onemolecule thick layer and form a chemical bond with a solid support. The TMDC surface is then brought into physical, non-covalent contact with the other side of the monolayer. The second one is based on the formation of self-assembled molecular networks $(S A M N s)^{25}$ on the TMDC surface itself, where the molecules often form a crystalline film, typically again one molecule thick.

Some of the first works in modifying TMDCs' properties through SAMs proposed the use of functionalized silanes. ${ }^{26}$ SAMs of alkyl silanes with functionalized chain-ends were formed on a $\mathrm{SiO}_{2}$ substrate onto which $\mathrm{MoS}_{2}$ flakes were transferred physically. Thus in this setup, the MoS 2 films interact with the end-groups that stick out from the surface. As expected, the electrical properties of MoS 2 varied depending on the functional groups that interact at the interface. They also observed the effect silane monolayers have on a p-doped substrate $\left(\mathrm{WSe}_{2}\right)$ is opposite to that on an n-doped substrate $\left(\mathrm{MoS}_{2}\right){ }^{27,28}$ SAMs of fluoroalkyltrichlorosilane and octyltrichlorosilane that have a positive dipole moment seem to accumulate electron carriers at the interface of TMDCs, shifting up the Fermi level in p-doped materials while shifting down the Fermi level in case of $n$-doped materials. This causes an inverse effect in threshold voltages of both types of materials. SAMs of 3-aminopropyltrimethoxysilane acted as $n$-doping agents by accumulating holes at the substrate interface, thus shifting down the Fermi level in p-doped materials and shifting it up in n-doped materials. These techniques also modulate the materials' work function as it directly depends on the Fermi level of the material. As previously seen in other systems, the magnitude of effects depends on the Fermi level of the material, the dipole moment of the molecule, and the strength of their interaction. 


\section{a)}
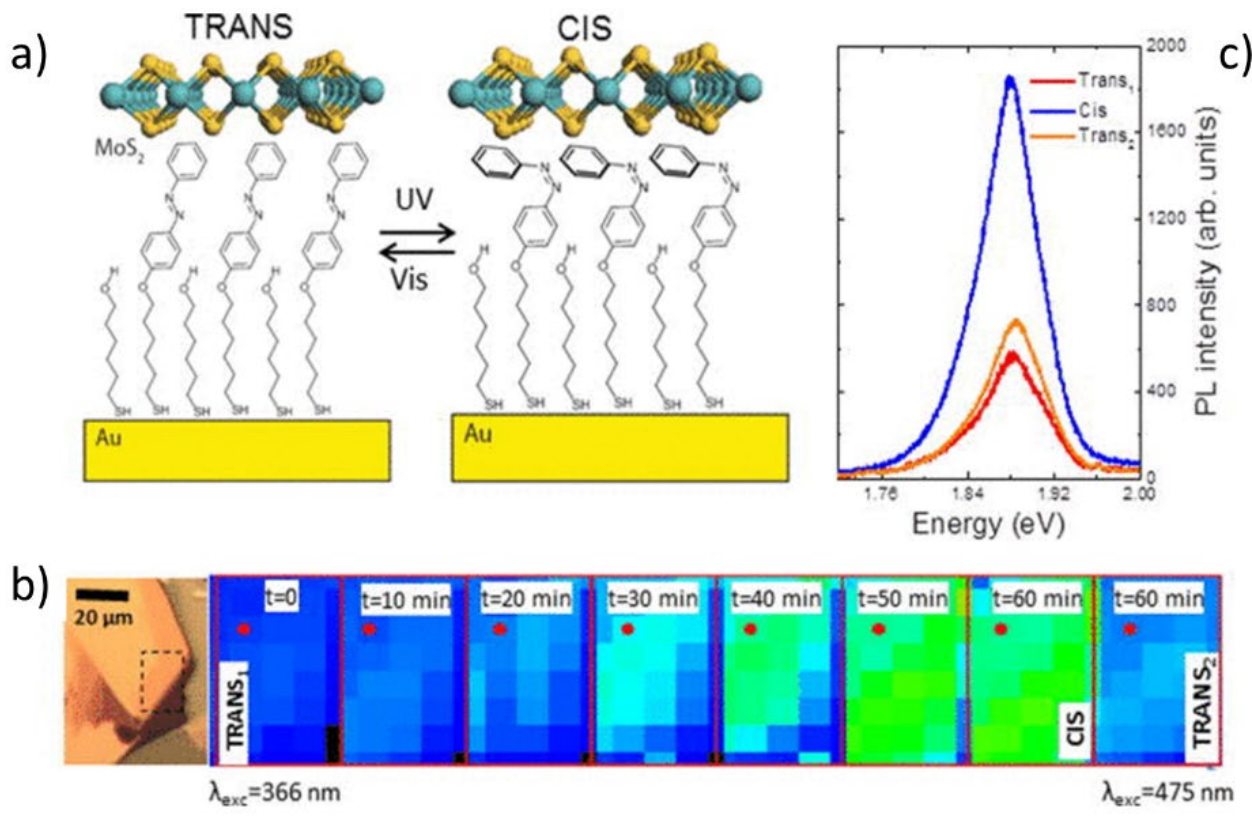

Figure 3. a) Scheme of a monolayer $\mathrm{MoS}_{2}$ sample gated in by trans- and cis-azobenzene. b) PL intensity recorded for 2L-MoS2 on the SAM after UV exposure for different times at the position of the red spot. Last map shows the switching back to trans after 120 min of white light exposure. C) PL spectra for 2L-MoS2 in trans (red), in cis (blue) and again in trans (orange). Reprinted with permission from reference 26. Copyright 2014 AIP Publishing.

Another interesting approach of tuning properties of TMDCs has come via organic molecules that interact through heteroatoms (i.e. nitrogen) and pi clouds. Azobenzene derivative SAMs represent one of such systems explored so far. ${ }^{29}$ Azobenzene molecules are interesting due to their cis-trans photoisomerization (Figure 3). The trans isomer can be converted into the cis form by UV radiation. The process is also photoreversible and turns from cis to trans under visible light, making it a photo-controllable system. Azobenzene is electropositive and the large contact potential in its trans state imparts an n-doping to the $\mathrm{MoS}_{2}$. Among the isomers, the trans form has a larger contact potential on interacting with the $\mathrm{MoS}_{2}$ surface than the cis form which leads to the higher doping impact of the former isomer. ${ }^{30}$ The photoisomerization from trans to cis lowers the magnitude of doping and restores some of the intrinsic properties. It is shown that the magnitude of this doping can additionally be controlled by using mixed selfassembled monolayers (mSAMs) where the proportion of azobenzene units per area can be modulated. It is shown that the type of doping can also be controlled by varying other chemical species present in the SAM. A system populated with electronegative species such as chlorine can exert the opposite effect and act as a p-type dopant. This makes MoS 2 on azobenzene systems a conveniently tunable semiconductor with photo-switchable doping levels.

Other systems that interact with $\mathrm{MoS}_{2}$ through heteroatoms and pi clouds are perylene derivatives, porphyrins, and phthalocyanine derivatives. Unlike silanes and azobenzene derivatives, these systems interact through SAMNs at MoS2 surface rather than by means of SAMs on a substrate like in the former. Perylene based dyes are excellent optoelectronic dopants due to their thermal and photo stability and have 
been used as n-type dopants in solar cells. ${ }^{31}$ Porphyrins display excellent molar absorptivity the order of $10^{6} \mathrm{M}^{-1} \mathrm{~cm}^{-1}$. Besides their high photo stability, porphyrins and phthalocyanines are popular due to their reduction potential that can be tuned by varying the metal centres and peripheric ligands. Metal phthalocyanines are also used in light harvesting systems where they act as both electron donors and electron acceptors to carbon nanotubes and graphene depending on their core metal. ${ }^{32}$ These metal centres also provide the catalytic nature that is advantageous in various applications.

SAMNs of perylene $\mathrm{N}$-alkyldiimides on $\mathrm{MoS}_{2}$ are explored as well. ${ }^{33}$ Deusberg and coworkers used a monolayer-thin film of perylene diimides (PDI) to facilitate atomic layer deposition (ALD) at TMDCs surfaces. ${ }^{34}$ What makes it an important development is that ALD is a highly precise deposition technique that is used to print gate oxides and integrated circuits on semiconductor surfaces. But the common reaction for ALD needs surface hydroxy groups whose absence on TMD surface made it challenging to develop TMD-based electronic devices. They used PDI modified with carboxy alkanes to introduce hydroxy groups at the TMD surface that acted as a base for the ALD reaction without modifying its pristine properties. PDI and porphyrin have also been used as n-type dopants to improve the photocurrent and photoresponsivity of $\mathrm{MoS}_{2}$ devices. ${ }^{35}$ Additionally, the thermal stability and strong interactions of these molecules can withstand the $1 \mathrm{~T}-2 \mathrm{H}$ phase transition in TMDCs. Thus, these molecules can even be used as non-covalent dopants in high-temperature applications. ${ }^{36}$ 

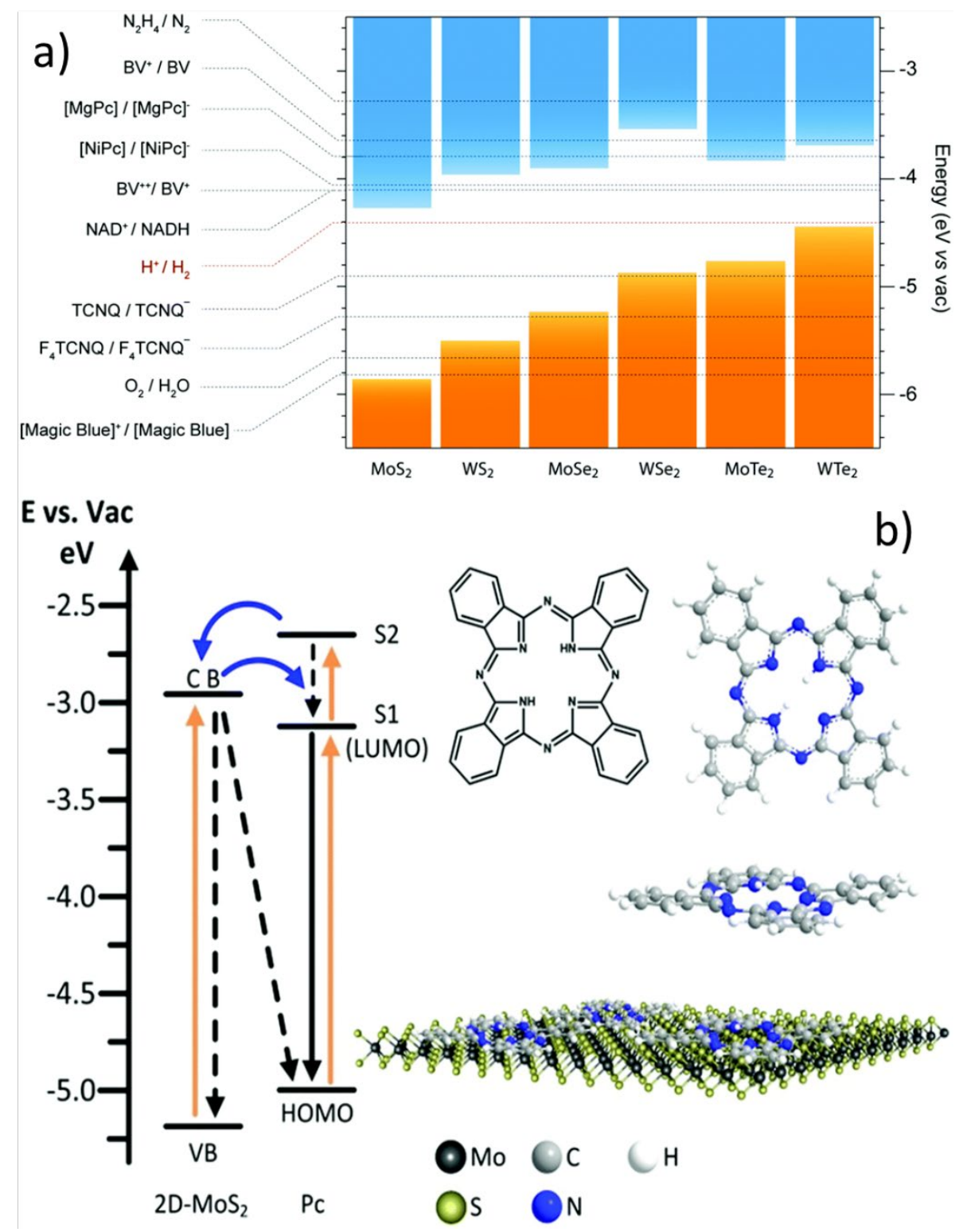

Figure 4. a) Comparison between the electrochemical redox potentials of various molecular dopants and the valence and conduction band edges of group-6-TMDs. Reprinted with permission from reference 12. Copyright 2018 The Royal Society of Chemistry. b) Energy diagram for $\mathrm{MoS}_{2}$ functionalized with phthalocyanine. Orange (excitation), black (radiative recombination) and blue (charge-transfer). Dashed lines show non-radiative processes. Reprinted with permission from reference 36. Copyright 2016 The Royal Society of Chemistry.

Phthalocyanines (Pc) are popular light-harvesting systems in solar cells and other optical applications due to their tunable HOMO-LUMO positions and bandgap that falls in the visible spectrum. ${ }^{37}$ Most common approaches to tune the properties of these systems are either by replacing the metal cores or by substituents on the Pc. Recently, Samori and co-workers showed that the electronic nature of phthalocyanines can also be tuned by on-surface functionalization of the metal centres of phthalocyanines assembled on $\mathrm{MoS}_{2}{ }^{38}$ The forced orientation and charge transfer in the SAMs of metallic phthalocyanines 
(MPc) at TMDC surface was taken advantage of to carry out efficient, unidirectional ligand functionalization by pyridine derivatives at the metal centres. This approach provided an additional tunability dimension to properties of the molecular system. Phthalocyanines are also interesting for photo-controllable doping. In the previous section, we discussed the photoswitchable doping in azobenzene functionalized MoS 2 devices. Phthalocyanines offer a different mechanism of control. These species have dual emission; between HOMO and LUMO termed as S1, and between HOMO and LUMO+1 termed as S2 emission (Figure 4). It happens that the conduction band minima of MoS 2 lay between the LUMO and LUMO+1 levels in metal-free phthalocyanine. In this scenario, phthalocyanine acts as an n-type dopant or a p-type dopant depending on the excitation wavelength. But this possibility is not practically realized due to an unfavourable charge transfer from the dye's S1 state. ${ }^{39}$ Nevertheless, phthalocyanines are important functionalizing systems for 2D materials with high potential in optoelectronic applications.

Functionalization and doping of $\mathrm{MoS}_{2}$ is not limited to the above-mentioned systems. In general, dye materials have their HOMO-LUMO transitions in the visible range and hence absorb in this range, coinciding with the bandgaps of semiconducting TMDCs. Spatial interaction of these molecules with TMDCs allows charge transfer between these systems that leads to a manipulation of Fermi levels and subsequent outcomes on electronic properties. Due to this reason, various dyes like 2,3,5,6-tetrafluoro-7,7,8,8tetracyanoquinodimethane (F4TCNQ), 7,7,8,8-tetracyanoquinodimethane (TCNQ), nicotinamide adenine dinucleotide (NADH), ${ }^{40}$ benzyl viologen (BV), ${ }^{41}$ methyl orange (MO), rhodamine 6G (R6G), and methylene blue $(\mathrm{MB})^{42}$ have been explored as dopants for TMDCs. This is not a comprehensive list, but an illustration of the array of possible dopants. Other functional molecules include triphenylphosphine, which was demonstrated to have air/water stable and reversible n-type doping-dedoping interactions with $\mathrm{MoS}_{2}{ }^{43}$ and oleylamine for which a decrease in contact resistance was reported. ${ }^{44}$
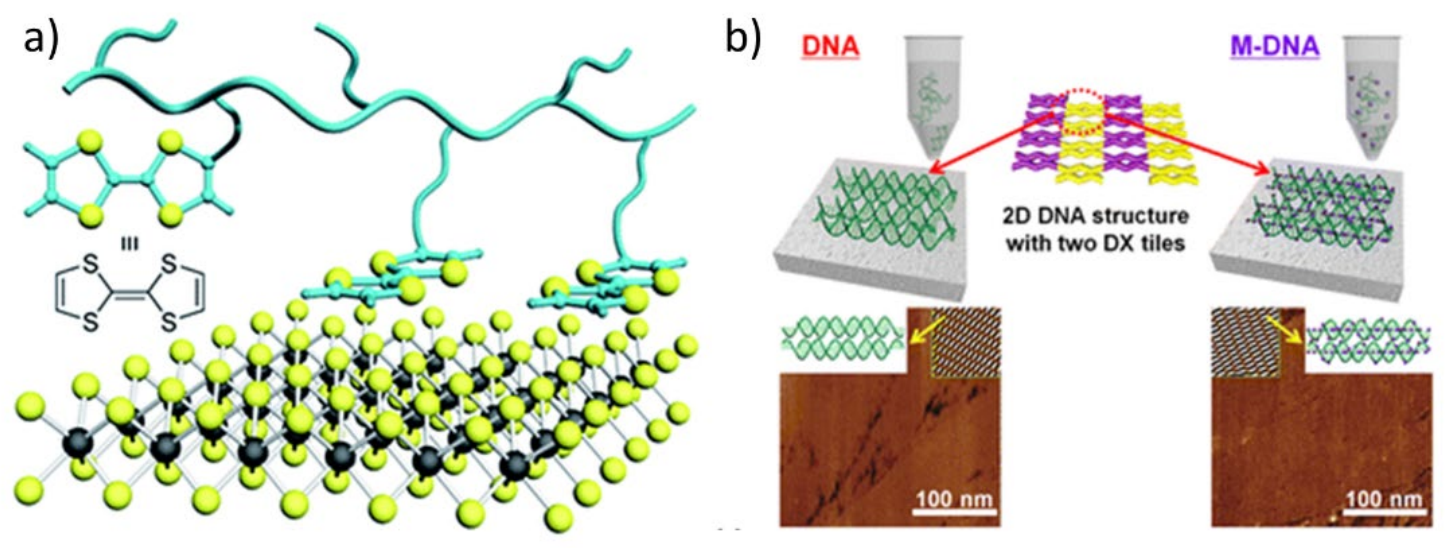

Figure 5. a) Schematic illustration of the physisorption of TTF on $\mathrm{MoS}_{2}$. Reprinted with permission from reference 42. Copyright 2016 Royal Society of Chemistry. b) Schematic diagrams of the fabrication of DNA and M-DNA DX structures, AFM images of pristine DNA and M-DNA with $4 \mathrm{mM} \mathrm{Cu}^{2+}$. Reprinted with permission from reference 43. Copyright 2014 American Chemical Society. 
Apart from small organic molecules, larger molecules could also be used to modulate TMDCs' properties through specific interactions. Polymers have been used for this purpose. Emrick and co-workers reported on polymers with tetrathiafulvalene (TTF) units in the sidechains as anchor group for noncovalent functionalization of $\mathrm{MoS}_{2}$ (Figure 5a). ${ }^{45}$ TTF acts as an n-type dopant. The polymer chain increases the dispersibility of $\mathrm{MoS}_{2}$ sheets in solvents. DNAs are another type of large molecules that have been used to functionalize TMDCs. Park and co-workers reported the self-assembly of DNA on $\mathrm{MoS}_{2}$ and WSe2 (Figure 5b) where the phosphate groups in the backbone of the DNA act as anchoring groups as well as $n$-dopants due to their electron rich nature. ${ }^{46}$ They also induce $p$-doping and tune the contact potential by means of DNA modified with metal ions (M-DNA). In this case, the metal ions withdraw the charge from the phosphate groups and reverse the effect. DNAs also present specific interactions that are useful in targeted sensing. Joo and co-workers reported the assembly of probe DNA ( $p$-DNA) modified with a fluorescent dye, Cy3, at WSe $\mathrm{W}_{2}$ surface as p-type dopants. ${ }^{47}$ They displayed the use of specific interactions of DNA as a tool for biosensing target DNA (t-DNA) indirectly through the photoluminescence intensity of WSe2/Cy3/p-DNA system. The PL intensity of the system increases with the binding of t-DNA because of de-doping of WSe 2 as DNA forms double strands. Though still in its infancy, the DNA-based functionalization methods will become more important in the future. Techniques such as DNA origami that allow programmed folding and scaffolding of DNA can be used to design systems that allow non-arbitrary functionalization of $\mathrm{MoS}_{2}$ surfaces, which could be switched between n-type, p-type, and dedoped selectively.

Self-assembly-based noncovalent functionalization of 2D materials is a rapidly growing field. We have summarized here various approaches that use self-assembly to functionalize TMD materials and tuning their semiconductor applications and optoelectrical properties. Although in this section we have focused on self-assembly processes, other approaches for thin film formation on TMDCs have been reported as well. $8,48-50$

\subsubsection{OTHER CONDENSED PHASE TECHNIQUES}

In this section, we give an overview of other condensed phase molecular functionalization techniques for functionalizing TMDCs that do not fall under the category of self-assembly. 
a)

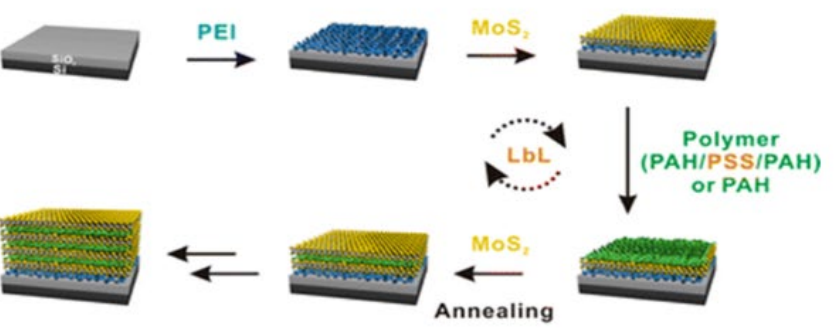

b)

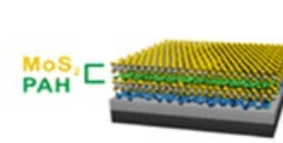

$2 \mathrm{M}_{\mathrm{si}}$

c)

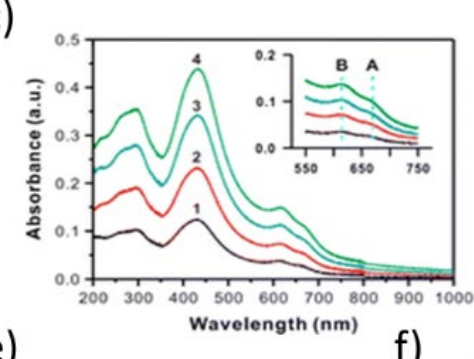

e)

f)

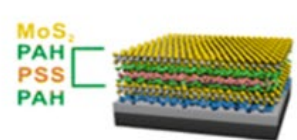

$2 \mathrm{M}_{\mathrm{n}}$

d)

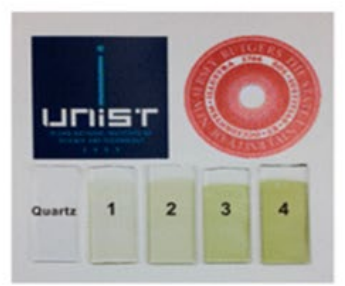

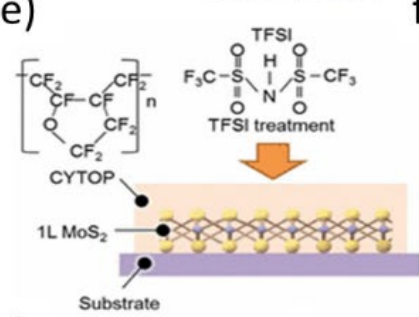

g)
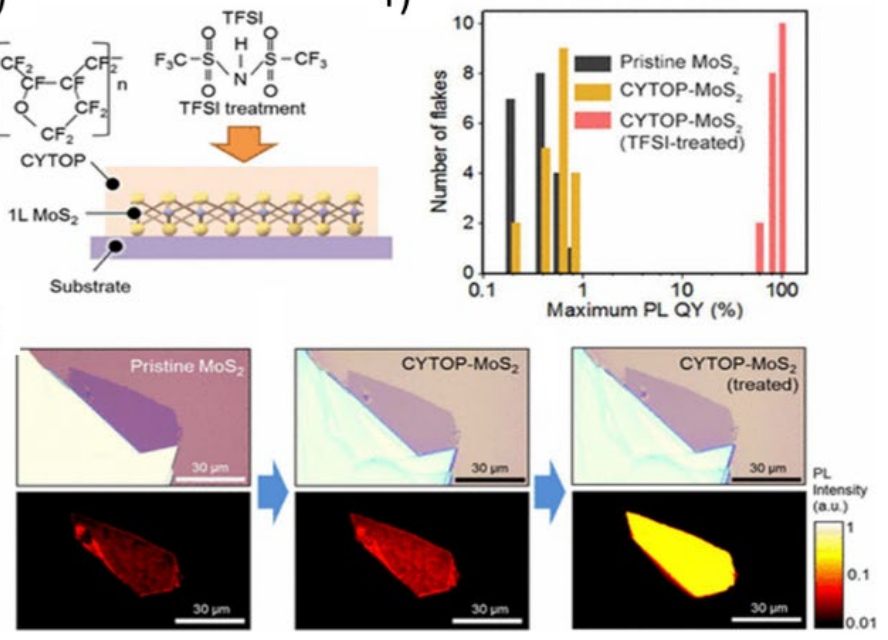

Figure 6. a) Schematic representation of the layer by layer assembly of $\mathrm{MoS}_{2}$ with polymer spacers. b) Scheme showing the $2 M_{S L}$ and $2 M_{T L}$. c) UV-vis absorbance spectra of the growth of $M_{0} S_{2}$ multilayers. d) Photographs of $M_{0} S_{2}$ multilayer films deposited on quartz slides with different number of layers. Reprinted with permission from reference 45. Copyright 2014 American Chemical Society e) Schematic of sample encapsulation/passivation showing a monolayer of $\mathrm{MoS}_{2}$ coated by CYTOP and treated by TFSI. f) histogram showing the PL quantum yield of 20 flakes after exfoliation (black), coating with CYTOP (yellow) and treatment by TFSI. g) PL images and corresponding optical micrographs for the three different cases. Reprinted with permission from reference 51. Copyright 2017 American Chemical Society.

Polymers are a common dielectric material for all kinds of semiconductors and have also been popular for applications in TMDC devices. Polymers are also used to encapsulate functional molecules at the TMDCs surface. The charged nature of TMDCs in solution makes them suitable candidates for multilayer assembly through techniques like Langmuir-Blodgett (LB) films $^{51}$ and layer-by-layer (LbL) assembly. ${ }^{52}$ Xie and coworkers prepared LbL films from a positively charged hybrid material composed by MoS 2 and poly(diallyl 
dimethyl ammonium chloride), and negatively charged poly(acrylic acid) (PAA). This material showed a good performance as functional separator in Li-S batteries.$^{53} \mathrm{MoS}_{2}$ and PAA in the membrane restrains polysulfide shuttling by impeding molecular diffusion and chemisorption. The membrane further improves cycle stability of Li-S batteries by regulating a homogenous Li-ion flux and hence suppressing Li dendrite formation. A similar approach was taken by Kim and co-workers who reported the LbL assembly of multilayered $\mathrm{MoS}_{2}$ films spaced by polymer films (Figure $6 \mathrm{a}-\mathrm{d}$ ). ${ }^{54}$ To this aim, the intrinsic negative charge of chemically exfoliated $\mathrm{MoS}_{2}$ (ce-MoS2) was used for the LbL deposition rather than introducing charge through a polymer hybrid. Polyallylamine hydrochloride (PAH), a positively charged polymer was used in the process as its counter-layer and a spacer for the next layer. The spacing could be increased by an interlayer of a negatively charged polymer, polystyrene sulfonate (PSS), resulting in a PAH/PSS/PAH spacer. Apart from acting as spacer, PAH also acts as a p-type dopant. They observed that films containing the three-layer spacer show improved PL intensity over a single layer spacer as a result of better quenching of the interlayer coupling. Similar results were also obtained by Wang and co-workers where they observed a $\mathrm{PL}$ enhancement and preservation of direct bandgap in twisted bilayer $\mathrm{MoS}_{2} .{ }^{55}$ Residual poly(methyl methacrylate) (PMMA) present between layers from the transfer process here preserves the direct bandgap of $\mathrm{MoS}_{2}$. MoS encapsulated with a thin film of PMMA has been shown to act as ambipolar transistor. ${ }^{56}$ A commercial polymer, cyclic transparent optical polymer, abbreviated as CYTOP, is an amorphous fluoropolymer with environmental stability and high optical transparency that is another interesting material. By virtue of its inertness, the CYTOP interlayer prevents unintentional doping of TMDCs by external species and substrate through Pauli blocking and carrier screening. ${ }^{57}$ It provides an ideal platform to study the intrinsic electrical and optical properties of 2D materials. MoS 2 and WS 2 displayed highly stable, near unity PL quantum yield after encapsulation with CYTOP in combination with treatment of superacid bis(trifluoromethane)sulfonamide to supress defects (Figure $6 \mathrm{e}-\mathrm{g}$ ). ${ }^{58}$

Other important and interesting contributions regarding the polymer-stabilized exfoliation of $\mathrm{MoS}_{2}$ and other TMDCs using polyvinylpyrrolidone (PVP) have been reported. ${ }^{59}$ Zhang and coworkers described for the first time the use of PVP as stabilizing agent. ${ }^{60}$ They showed a facile method to prepare stable exfoliated few-layer $\mathrm{MoS}_{2}$ in aqueous as well as many organic solvents. They expanded this approach to other TMDCs and achieved a narrow size distribution ${ }^{61}$ improving their method by using the previously successful combination of grinding and ultrasonication. 62 Based on these results, Wang and coworkers obtained the highest yield of a polymer-based exfoliation using a coupled ultrasonication-milling process. ${ }^{63}$

Apart from PVP, biopolymers like proteins bovine serum albumin (BSA ${ }^{64,65}$ and gelatin ${ }^{66}$ have also been used to stabilize exfoliated TMDCs in aqueous solutions. Hydrophobins, which are small proteins made of few hundreds of amino acids, were also used to exfoliate and stabilize TMDCs. These proteins interact through hydrophobic interactions between their domains and the surface. The hydrophilic part of these proteins forms an interacting link between the particles and aqueous solution. ${ }^{67}$ Apart from stabilizing these colloids, the protein-TMDC interactions also improve the physiological stability and biocompatibility of these particles. The above properties combined with high absorption of TMDC particles in near-IR range 
have encouraged researchers to use functionalized TMDCs for various therapeutical applications including photothermal therapy of cancers and photoimaging of tumours. ${ }^{65,68}$

This hydrophobic interaction between TMDC and proteins was also exploited for biosensing of prostate specific antigen (PSA). Anti-PSA antibodies were dispersed on the surface of an MoS transistor sensor. When exposed to different concentrations of PSA, increasing antibody-antigen interaction emerges in the n-type device as a corresponding decrease in off-state current under negative bias. ${ }^{69}$ The sensor could detect concentrations as low as $1 \mathrm{pg} / \mathrm{mL}$, which is a few orders of magnitude below the clinical cut-off level for PSA.

Other studies of polymer-TMDC interactions report properties such as increased photoresponsivity, ${ }^{70}$ and improved mechanical and thermal stability ${ }^{71}$ of $\mathrm{MoS}_{2}$ by polymer encapsulation.

For finishing this section, we should remark that even if we have focused the discussion on the relatively popular group of TMDCs, there is important literature concerning other 2D materials including hBN, ${ }^{72,73}$ phosphorene, ${ }^{74}$ or MXenes. ${ }^{75}$ The next section will follow discussions on covalent functionalization protocols for 2D materials.

\section{Covalent FunctionalizATION OF 2D MATERIALS}

The covalent functionalization of 2D materials has been deeply explored for graphene and related carbonbased materials with special focus on the use of highly reactive radicals (diazonium compounds, carbenes, etc.) or cycloaddition reactions. ${ }^{76,77}$ Nevertheless, although some of these methodologies can be extended to the modification of other 2D materials, in some cases their specific reactivities require new methods for their covalent functionalization. ${ }^{77}$ Taking the $\mathrm{MoS}_{2}$ case as example, the most obvious option for covalent functionalization would be the formation of a covalent bond between the molecular modifier and the sulfur atoms of the surface. Indeed, the first reported chemical modification using covalent chemistry on this material consisted of the ligand conjugation of a thiol moiety on defects formed during the chemical exfoliation of bulk MoS2. ${ }^{78}$ After the publication of this report, the chemical functionalization has been widely explored for the highly reactive metallic phase $1 \mathrm{~T}-\mathrm{MoS}_{2}$ and $\mathrm{MoS}_{2}$ with a considerable number of defects and sulfur vacancies. Nevertheless, the application of these approaches to functionalize pristine surfaces is not straightforward due to the inherent inertness of the basal plane of MoS2. Therefore, new strategies for the robust functionalization of the basal plane of $2 \mathrm{H}-\mathrm{MoS}_{2}$ have been proposed, for instance using electrophilic compounds such as maleimides or organohalides. In this section, we summarize the use of different precursors for the modification of defective/pristine TMDCs: 1) metal complexes to generate coordinate bonds; 2) sulfur-containing species; 3) organohalides; 4) radicals generated through diazonium chemistry and 5) maleimides. (Figure 7) 


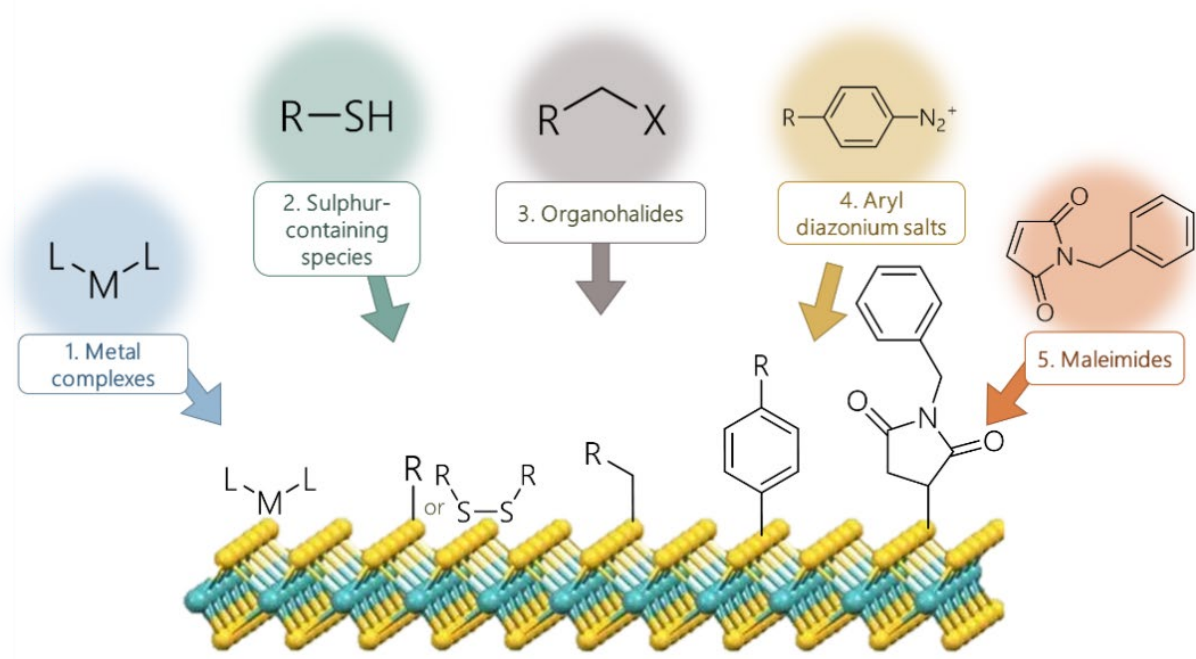

Figure 7. Schematic representation of the different molecular chemistry approaches that have been proposed in the last years for covalent functionalization of 2D TMDCs.

\subsection{MetAL COMPLEXES}

Metal complexes can be used as a bridge for the functionalization of layered TMDCs such as MoS2. This strategy was previously shown to be successful for the modification of graphene oxide. ${ }^{79}$ Later, it was extended to $\mathrm{MoS}_{2}$ nanosheets in order to form MoS 2 paper cross-linked with polymeric ligands by adding divalent metal ions. ${ }^{80}$ The basis of this methodology is the interaction of the divalent metal ion ( $\mathrm{Cu}^{2+}$ ions) with the sulfur atoms on the surface and the simultaneous coordination of this ion to the carboxylate $(\mathrm{C}=$ O) groups of the polymer ligand. The formation of this bond was confirmed by several techniques such as UV-vis, FTIR or XPS. With this method, $\mathrm{MoS}_{2} /$ PMMA nanocomposites with enhanced mechanical properties were obtained.

A systematic study of the interaction of liquid-exfoliated $\mathrm{MoS}_{2}$ sheets with acetate salts with the general formula $\mathrm{M}(\mathrm{OAc})_{2}\left(\mathrm{M}=\mathrm{Ni}, \mathrm{Cu}, \mathrm{Zn} ; \mathrm{OAc}=\right.$ acetate) was carried out by Backes and coworkers. ${ }^{81}$ (Figure 8a) The sheets of $\mathrm{MoS}_{2}$ were exfoliated using 2-propanol (IPA) as a solvent which stabilizes the $2 \mathrm{H}-\mathrm{MoS}_{2}$ phase. The formation of a coordination bond of the metal center with the sulfur could be confirmed by XPS measurements. The appearance of a second component in the S $2 p$ core level spectra at higher energies in the functionalized materials is in line with a different chemical environment due to the coordination of the metal ion. The degree of functionalization depends on the chemical nature of the metal ion due to the different affinity of the ions to bind the sulfur atom. The higher efficiency is obtained in the case of Cu (50\% of the $\mathrm{S}$ of the surface for $2 \mathrm{H}-\mathrm{MoS}_{2}-\mathrm{Cu}(\mathrm{OAc})_{2}$ ) when compared to $\mathrm{Ni}(30 \%)$ and $\mathrm{Zn}(10 \%)$ (Figure $8 \mathrm{~b}$-d). The main advantage of this functionalization method is the improvement in the dispersibility of the material in a wide range of solvents, with different polarities. 

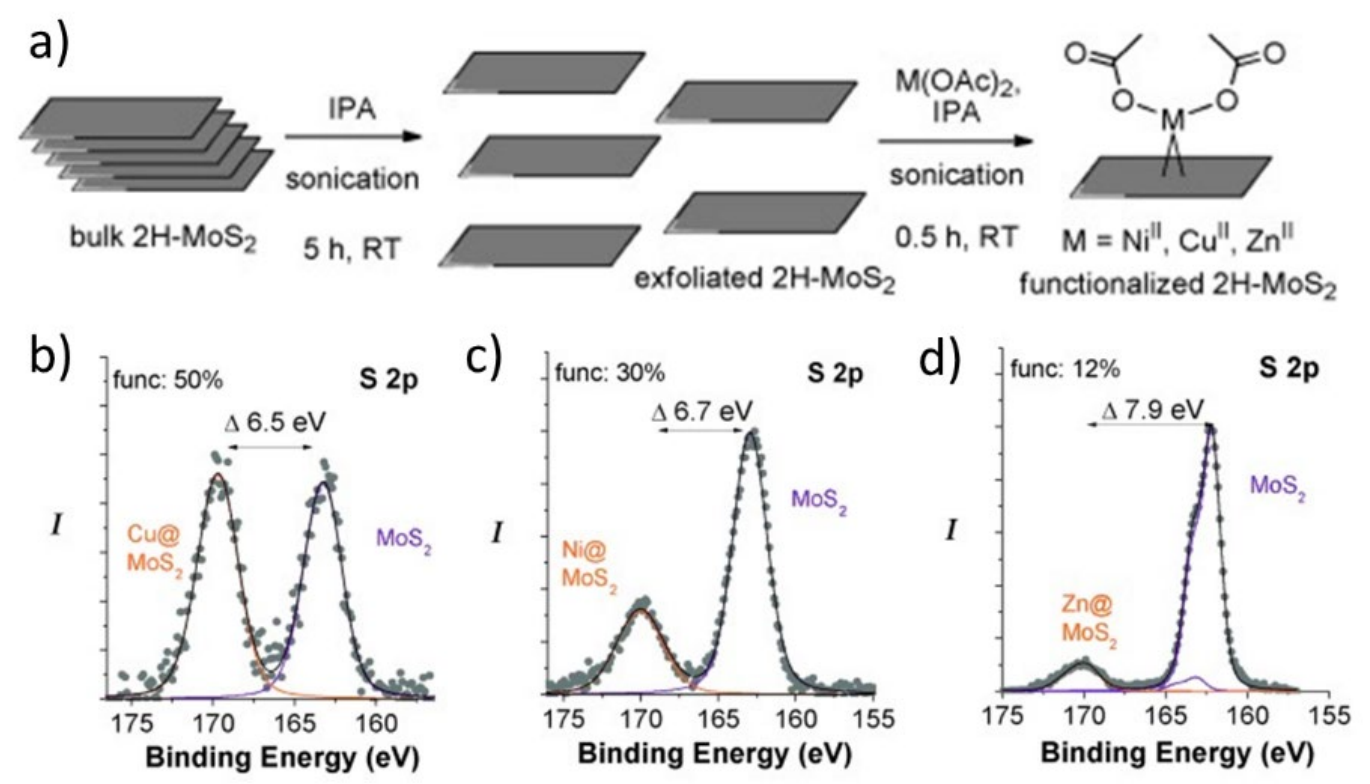

Figure 8. a) Schematic representation of the exfoliation and functionalization of $2 \mathrm{H}-\mathrm{MoS}_{2}$ using metal acetate salts (IPA $=2$-propanol). b-d) Fitted XPS high resolution spectra for the S2p core level spectra using different metals in the acetate salt showing the different reactivity found depending on the metal. Adapted with permission from reference 57. Copyright 2015 Wiley-VCH.

\subsection{SULPHUR-CONTAINING SPECIES}

The use of thiols to functionalize metal surfaces with SAMs has been thoroughly described in the literature. ${ }^{82}$ This approach is based on the formation of a covalent bond between the sulphur atom and the metallic atom of the surface due to a specific molecule-substrate bond. The absence of this specific interaction impedes that thiols can be used in the covalent functionalization of 2D materials such as graphene, although some authors have suggested the formation of this covalent bond on graphite surfaces as well. ${ }^{83}$ However, in the case of $\mathrm{MoS}_{2}$, the functionalization can be achieved by conjugation of thiols at the sulphur vacancy sites or defects.

The early experiments using thiol chemistry for the modification of $\mathrm{MoS}_{2}$ were carried out on chemically exfoliated $\mathrm{MoS}_{2}$ flakes. ${ }^{78}$ The molecular functionalization of the surface was evaluated through the shift in

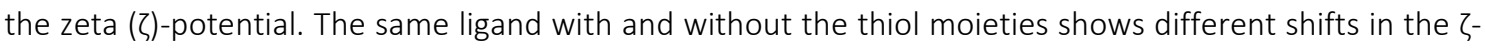
potential, confirming the role of the thiol group. The material was further characterized by FTIR to check the presence of the functional groups on the surface. The electronic properties of the MoS $\mathrm{M}_{2}$ flakes functionalized with thiol molecules were explored as well. ${ }^{84}$ Modified surfaces were characterized using XPS, photoelectron spectroscopy in air (PESA) and PL experiments. The results show a shift in the conduction and the valence bands for the functionalized materials, which was explained by considering the mild Lewis base character of the thiols, leading to an overall electron donating effect. PESA experiments showed a cathodic shift of both bands up to $500 \mathrm{meV}$, although no changes in the band-gap were observed. XPS confirmed changes in the Fermi level depending on the chemical nature of the thiol used (aromatic or aliphatic). Although the interaction between the thiol and the MoS 2 surface was assumed to be covalent, some doubts about the real nature of this bond emerged later. ${ }^{85}$ In this case, the functionalization was 
targeted with the use of cysteine. The results showed that the oxidation of cysteine to the disulphide cysteine during the functionalization can take place on the $\mathrm{MoS}_{2}$ surface. The presence of this oxidised molecule on the modified surface was confirmed through XPS and diffuse reflectance infrared spectroscopy (DRIFT) spectra. Both techniques showed the characteristic signals for the disulphide product. In a recent theoretical study, this discrepancy was explored in detail. ${ }^{86}$ The calculations showed that the dimerization process which leads to the physisorbed material is thermodynamically favoured when $\mathrm{S}$ adatoms are present on the surface. However, when there are sulphur vacancies, these disulphide species can be reduced back to the thiol form and anchor on the surface defect to repair these vacancies.

Recently, new insights into the functionalization of defective $\mathrm{MoS}_{2}$ surfaces using organic thiols have been gained. ${ }^{87}$ In this article, the authors argued that the attachment of organic moieties such as thiophenols occurs on the defects of the surface forming a covalent bond. By tuning the functional group of the molecule, the functionalization density can be controlled. In this sense, the highest degree of functionalization is obtained for the $\mathrm{NO}_{2}$-substituted molecules and the lower with the $\mathrm{NH}_{2}$-sustitued ones. These results show the influence of the functional group on the efficiency of the modification and how the choice of the modifier can be used to control the number of vacancies or defects on the material. Interestingly, a spectroscopic indicator to monitor and quantify the degree of functionalization was discovered. The intensity of the $2 \mathrm{LA}(\mathrm{M})$ normalized peak to $\mathrm{A} 1 \mathrm{~g}$ peak correlates to the degree of functionalization and, therefore to the number of defects, being of special interest in the field of the molecular functionalization of $\mathrm{MoS}_{2}$ (Figure 9, left panel). 


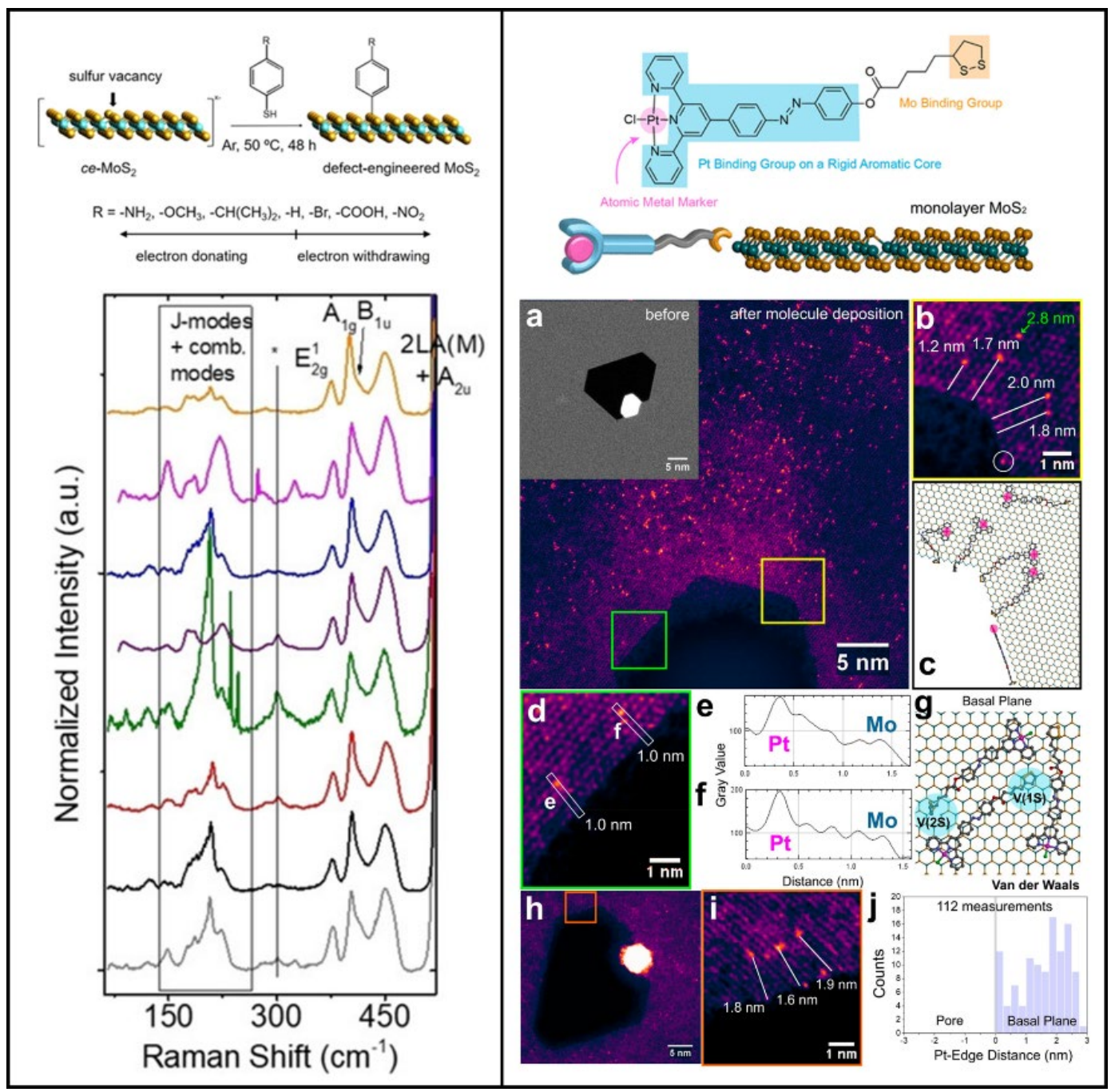

Figure 9. (left panel) Schematic illustration of the functionalization of $\mathrm{MoS}_{2}$ sheets using thiophenols by using defectengineering. Raman spectra corresponding to the functionalized material (grey = non-functionalized; black $=\mathrm{NO}_{2} ;$ red $=\mathrm{COOH}$; green $=\mathrm{Br}$; purple $=\mathrm{H}$; blue $=$ Pro; magenta $=\mathrm{OMe}$ and orange $=\mathrm{NH}_{2}$ ). Reprinted with permission from reference 63. Copyright 2020 Wiley-VCH. (right panel) ADF-STEM images of Pt-marked molecules and detailed study on how the molecule anchors to the surface. Reprinted with permission from reference 66. Copyright 2020 American Chemical Society

The effect of the incorporation of thiol moieties on the electrocatalytic performance of $\mathrm{MoS}_{2}$ surfaces has been studied as well. ${ }^{88}$ The results prove that the surface functionalization of the basal plane of monolayers of $\mathrm{MOS}_{2}$ leads to a decrease in the number of active sites for the hydrogen evolution reaction (HER), showing a passivation of the material toward this reaction. In the same article, the possibility of building heterostructures in a second step after the modification was demonstrated by incorporating PbSe quantum dots on a surface modified by dithiol molecules.

Regarding the sulphur chemistry, some studies show the possibility of using other molecules containing sulphur-units such as dithiolanes ${ }^{89,90}$ or dithiolenes for the functionalization of $\mathrm{MoS}_{2} \cdot{ }^{91}$ It has been proven that the covalent functionalization using 1,2-dithiolanes derivatives occurs preferentially at the sheet edges 
of $\mathrm{MoS}_{2}{ }^{89}$ Recent results using annular dark-field scanning transmission electron microscopy (ADF-STEM) have helped to gain insight into the attachment of these units to the surface. ${ }^{90}$ For these experiments, a specific molecule containing a single transition metal atom (Pt) was designed in order to track the binding of the molecules on the material. The results confirmed that the covalent attachment occurs to the edges and the surface around pores on the $\mathrm{MoS}_{2}$ surface. Importantly, with this technique it was possible to determine the conformation and the orientation of the molecules once they are bound to the edge (Figure 9 , right panel). The interaction of the rest of the molecule with the basal plane of MoS 2 has been proved, showing van der Waals interactions between the aromatic core and the basal plane of the material. These results show the influence of the van der Waals interactions with the material after the covalent bond is established. The covalent attachment of dithiolanes has been used as a platform for further postfunctionalization. ${ }^{92}$ In this case, the surface of exfoliated $M_{0} S_{2}$ and WS is functionalized with 1,2dithiolane-BOC followed by acidic deprotection of the covalently anchored organic moieties. After this second step, the surface contains charged ammonium units exposed to the solution which can interact with porphyrin molecules via electrostatic interactions due to the negative charge of the carboxylatesubstituted porphyrin. Time-resolved photoluminescence experiments have shown that the transduction of energy from the photoexcited porphyrin to the exfoliated material takes place in a donor-acceptor process.

Thiol chemistry has been explored as an option to form robust interfaces between other 2D materials and complex molecules such as porphyrins, with a well-known activity toward electro- and photocatalysis. Thiolated tetraphenyl porphyrins have been attached covalently to the surface of chemically exfoliated $\mathrm{MoSe}_{2}$ nanosheets. ${ }^{93}$ The formation of a covalent bond was confirmed by using XPS, where the formation of a sulfo-selenide bridge can be detected. To fully understand the formation of this bond between the thiolated porphyrin and the MoSe 2 additional DFT calculations were carried out. In these calculations, only the adsorption of the thiolated alkyl chain (propanethiol) was considered given that ionic and van der Waals interactions are weak and the physisorbed material is removed after the washing protocol. Results show that the adsorption of the thiol on the basal plane of the material does not lead to an attractive interaction, which means no covalent bond is formed. Nevertheless, at the edge of the MoSe2, the energy of adsorption is $-28.8 \mathrm{kcal} \mathrm{mol}^{-1}$, a much favoured interaction and, consequently, the formation of a covalent bond is possible. The functionalized nanosheets were further evaluated for their use in the electrocatalysis and photocatalysis of a model reaction such as the HER reaction. The hybrid materials showed an improvement in the photoelectrocatalytic activity with a shift in the onset potential for the HER reaction and a relative light enhancement (\% of overpotential decrease due to the light illumination) 4 times higher than for unmodified $\mathrm{MoSe}_{2}$.

\subsection{ORGANOHALIDES}

The functionalization of monolayers of different TMDCs (MoS $2, \mathrm{WS}_{2}$ and $\mathrm{MoSe}_{2}$ ) can be achieved with the use of organohalides. ${ }^{10}$ This reaction can proceed spontaneously if the highly reactive $1 T$ phase of these materials, obtained after their treatment with n-BuLi from the $1 \mathrm{H}$ phase, is used and it is based on the 
direct electron transfer between this $1 T$ phase and the organohalide molecule. In this way, advantage was taken of the negative charge of the material after the chemical exfoliation with $n$-BuLi. The surface was treated with methyl iodide and 2-iodoacetimide. Chhowalla et al. indicated that the functionalization using this approach only occurs in the $1 \mathrm{~T}$ phase of the material and with an extension of around 30 atomic percentage (at\%) relative to the transition metal content. Interestingly, although scanning transmission electron microscopy (STEM) experiments show that the octahedral coordination of Mo atoms in the 1T phase was not altered, the electronic properties of this phase are profoundly affected from metallic to semiconducting. These changes in the optoelectronic properties are explained by an opening of the band gap which gives rise to the tunable photoluminescence of the material. Authors show that this strategy is applicable to CVD monolayer MoS2 with similar results. Raman and XPS results show that after the functionalization it is possible to recover the $1 \mathrm{H}$ phase with annealing at $\mathrm{T} \geq 300^{\circ} \mathrm{C}$ while maintaining the functional group on the surface as revealed by ATR-FTIR measurements.

The use of different para-substituted iodobenzenes have been explored as well for the functionalization of $\mathrm{MoS}_{2}$ and $\mathrm{MoSe}_{2}$ nanosheets. ${ }^{94}$ As mentioned before, the reaction takes place in the $1 \mathrm{~T}$ metallic phase of the materials leading to the cleavage of the $\mathrm{C}-\mathrm{I}$ bond and the formation of a C-S or C-Se bond. In this electron rich phase, the degree of functionalization can reach values around $24 \%$ for electron withdrawing substituents. Moreover, more complex molecules such pyrenes, coumarin or porphyrin derivatives were tested, showing the versatility of this protocol. The molecular functionalization of the $2 \mathrm{H}$ phase of both materials has been explored in a following step, showing a much lower reactivity and the need of using a $\mathrm{Pd}^{0}$ catalyst to promote the reaction. DFT calculations shed light on the interaction of the molecule with the different phases. For all the functional groups analyzed $\left(-\mathrm{OCH}_{3},-\mathrm{H}\right.$ and $\left.-\mathrm{NO}_{2}\right)$ the values for the binding energy in the $2 \mathrm{H}$ phase are smaller than for the $1 \mathrm{~T}$ phase. These results confirm that the functionalization using organohalides is much more efficient and stronger in the $1 \mathrm{~T}$ phase. The theoretical study of the band structure and the projected density of states show that after the functionalization, the valence band and conduction bands of the $1 T$ phase are shifted and a gap opens between them. Moreover, it has been proven that the work function strongly changes for the $1 \mathrm{~T}$ phase of MoS2. The work function for the pristine $\mathrm{MoS}_{2}$ surface $(5.09 \mathrm{eV})$ is reduced when $\mathrm{R}=-\mathrm{H}(4.46 \mathrm{eV})$ and $\mathrm{R}=-\mathrm{OCH}_{3}(4.67 \mathrm{eV})$ are used. In contrast, the use of $\mathrm{R}=-\mathrm{NO}_{2}$ implies an increase in the value of the work function to $5.22 \mathrm{eV}$ due to the electron-withdrawing nature of this functional group.

As mentioned earlier, the covalent functionalization of the $1 \mathrm{~T}^{\prime}$ phase of the $\mathrm{MoS}_{2}$ and related materials can be achieved by using alkyl halides such as methyl iodide. However, the main disadvantage is the relatively low efficiency that is obtained (around the 25-30\%). This low efficiency can be explained in terms of the dependence on the negative charge of the material for the direct electron transfer. To improve the grafting density using alkyl halides a new approach using the activation of the molecules by reducing species has been proposed. ${ }^{95}$ The reducing agents employed consisted of one-electron metallocene (nickelocene, cobaltocene, etc.) which can react with the corresponding alkyl halide to produce a radical that binds to the surface. XPS and ATR-FTIR experiments confirm the presence of the alkyl chains in the modified 
material. The results of XPS can be used as an estimation of the efficiency of the modification and the surface coverage. Importantly, these data show that the reductant-activated functionalization is highly efficient when compared with the modification without reducing agent. In the best case, the coverage can increase up to $70 \%$ with a significant increase of 1.5-2 times compared to the one obtained previously (Figure 10). The influence of the alkyl chain and the leaving group was evaluated as well. It has been shown that the best results are obtained for iodomethane. The grafting is not efficient when chloride is used as leaving group or when the molecule has a high steric hindrance such as for secondary and tertiary alkyl iodides. The use of reducing agents in combination with chemical exfoliation can be seen as a promising way of attaching alkyl chains by means of alkyl iodides to several TMDC materials.

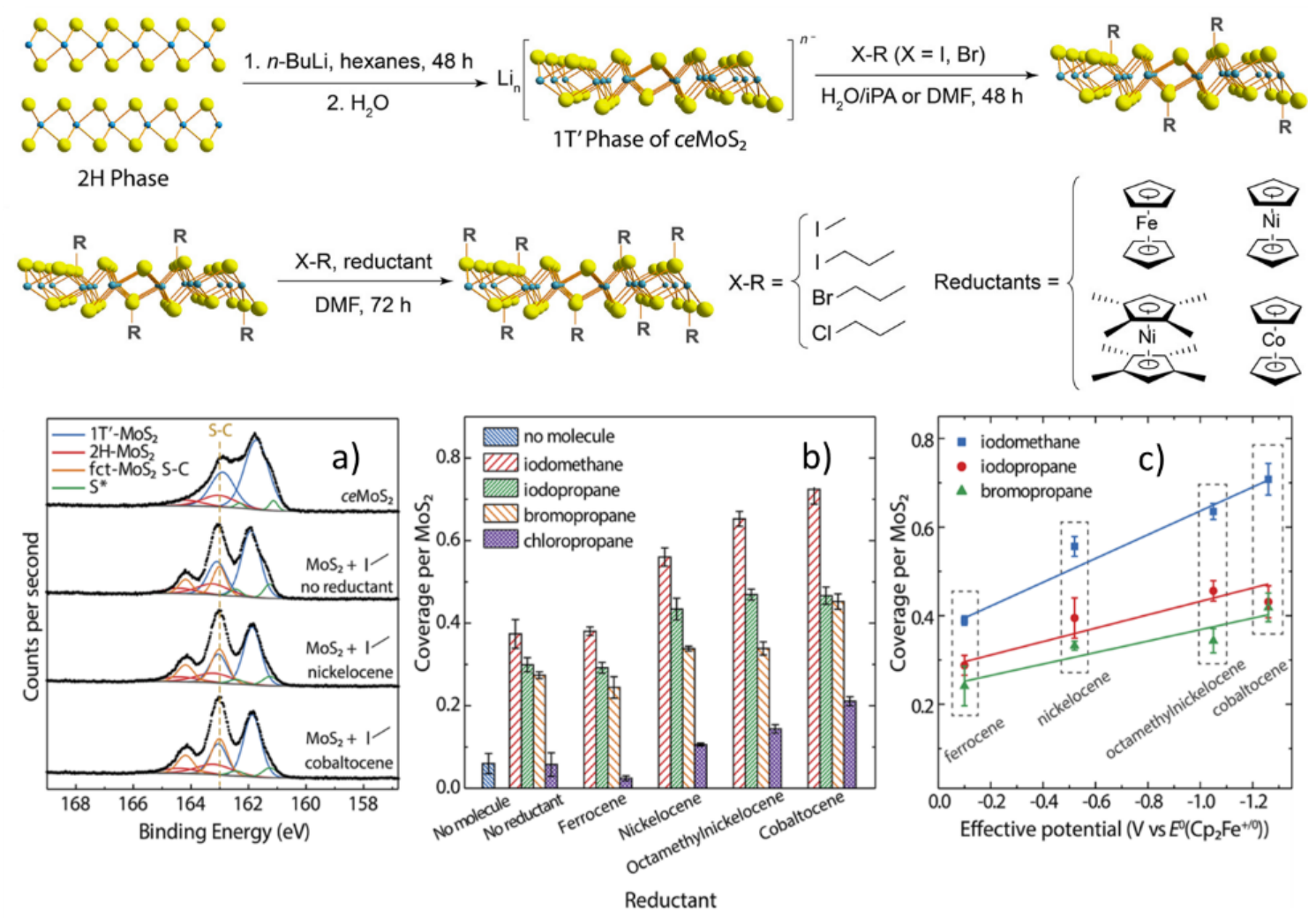

Figure 10. The functionalized $1 T^{\prime}-M_{0} S_{2}$ nanosheets are obtained by exfoliation in water and functionalized using alkyl halides (X-R). The functionalization can be promoted by adding one-electron metallocene reductants. a) XPS high resolution spectra of the $S 2 p$ region for the quantification of the coverage using the peak areas corresponding to the $S$ atoms. b) calculated coverages for different alkyl halides using diverse reducing agents. c) coverage per $\mathrm{MoS}_{2}$ unit depending on the effective potential of the reductants. Adapted with permission from reference 71. Copyright 2020 American Chemical Society.

\subsection{ARYL DIAZONIUM SALTS}

Aryl diazonium salts have emerged as the preferred reagents for the functionalization of a wide number of materials due to their high efficiency and versatility. The reaction consists of the reduction of the diazonium ions through a homolytic process leading to the formation of aryl radicals and the release of nitrogen. These aryl radicals are highly reactive and react with the surface forming a covalent bond between the modifier and the surface. Regarding the modification of 2D materials, diazonium ions have been shown to be very suitable for the covalent modification of both surface-supported graphene and graphene 
dispersions. ${ }^{96,97}$ The functionalization of these materials has been carried out by spontaneous decomposition of the aryl diazonium salt or by electrochemical or chemical activation. Raman spectroscopy has been chosen in most of the cases as a sensitive technique to detect the functionalization of graphene due to the characteristic $D$ band in the Raman spectra when the modification takes place. Given their success for the functionalization of graphene, the use of aryl diazonium salts has been extended for the functionalization of other 2D materials such as $\mathrm{MoS}_{2}, \mathrm{WS}_{2}, \mathrm{MoSe}_{2}, \mathrm{WSe}_{2}{ }^{98}$, black phosporous (BP) ${ }^{99}$ or hexagonal boron nitride $(\mathrm{h}-\mathrm{BN})^{100}$

The molecular functionalization of $\mathrm{MoS}_{2}$ using diazonium salts emerges as an alternative that does not require the presence of defects on the surface and can be used, as a consequence, to modify pristine surfaces of MoS2. Initial studies were done using chemically exfoliated MoS 2 by intercalation of $n$-butyl lithium, dispersion in water and ultrasonication. ${ }^{101}$ Once the negatively charged MoS 2 flakes are exfoliated, the aryl diazonium ions are incorporated and act as weak electrophiles. Interestingly, around $10-20 \%$ of the surface sulphur atoms are involved in the formation of a C-S bond. The covalent modification has been confirmed by TGA analysis, showing a mass loss in the temperature range of $220^{\circ} \mathrm{C}-450^{\circ} \mathrm{C}$ related to strongly bonded material. Indeed, it has been demonstrated that the annealing of the material at $350^{\circ} \mathrm{C}$ restores the pristine $2 \mathrm{H}-\mathrm{MoS}_{2}$. DFT calculations were performed to evaluate the $\mathrm{C}$-S bond. The value for the reaction energy at low coverages $\left(E_{\text {react }}=2.33 \mathrm{eV}\right)$ and the analysis of the Bader charges showing a partial negative charge on the $\mathrm{S}$ atoms confirm that, although physisorption cannot be excluded, the covalent reaction is clearly favored.

The aforementioned functionalization protocol has been applied to pristine MoS obtained by mechanical exfoliation from bulk MoS $2 .{ }^{102}$ The coverage dependence on both numbers or layers and the defects on the surface were evaluated. The AFM results show that if a pristine surface is used, the surface coverage of the deposited aryl diazonium salts is higher in the case of a bilayer of $\operatorname{MoS}_{2}$ when compared to the monolayer (Figure $10 \mathrm{a}-\mathrm{d}$ ). Some additional experiments in combination with DFT calculations showed that the S vacancies or defects can serve as nucleation centers in the initial stage of the functionalization. These calculations estimate that when an aryl group is attached on the surface, there is a charge reorganization and as a result, an increase in the reactivity of the surrounding area. It can be concluded that the growth of the layers is based on a cooperative surface reaction mechanism. Importantly, the semiconducting properties of the material are not disrupted as shown by the PL measurements. The reaction kinetics of this spontaneous functionalization of $\mathrm{MoS}_{2}$ using aryl diazonium salts was explored in later experiments using 4-nitrobenzene diazonium (4-NBD) tetrafluoroborate. ${ }^{103}$ Different adsorption isotherms were used to fit the dependence of the coverage on both time and concentration, extracted from AFM images (Figure 10 e-f). The results show that, studying the AFM images at different concentrations, the Freundlich and Temkin isotherms are the most suitable ones to describe the reaction kinetics of this system which include both the inhomogeneity of the $\mathrm{MoS}_{2}$ surface in terms of energy and the indirect adsorbate-adsorbate interactions with the already grafted nitrophenyl groups. Moreover, the dependence of the coverage with 
time shows that reaction kinetics can be described with a pseudo-second order model, confirming the interaction between 4-NBD moieties.

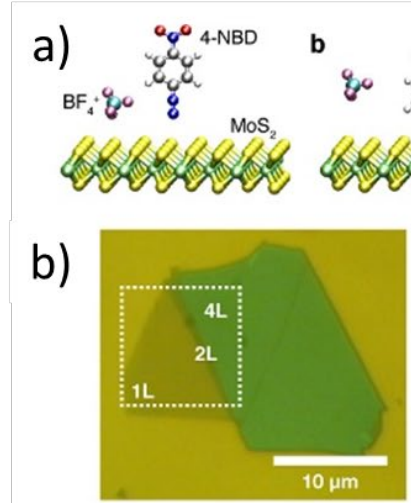

e)
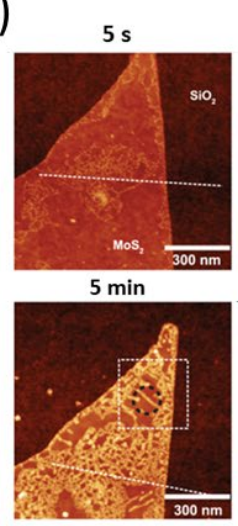

g)

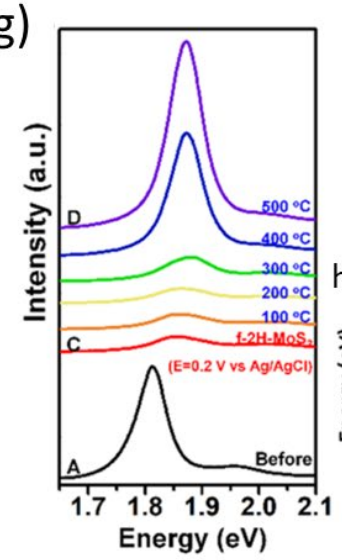

c)

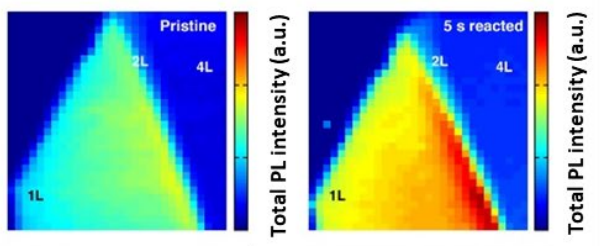

d)

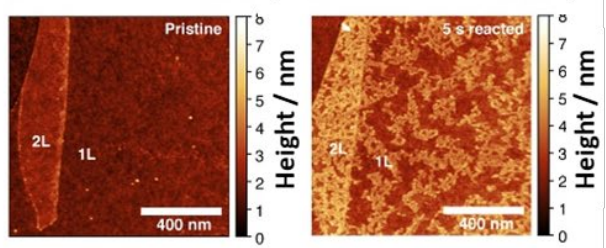

f)

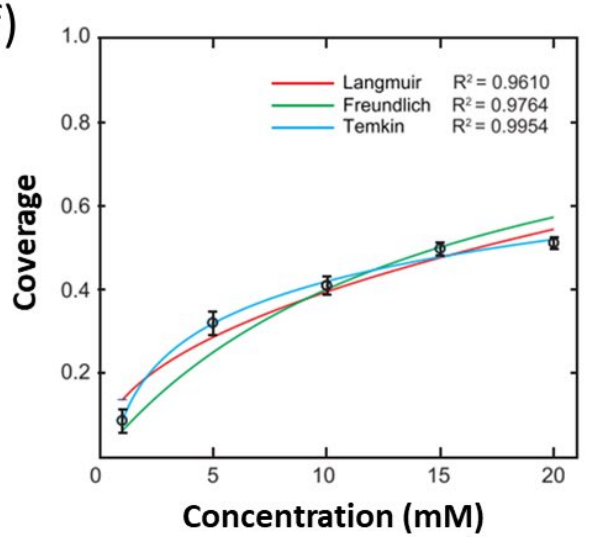

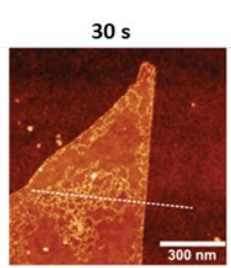

$10 \min$

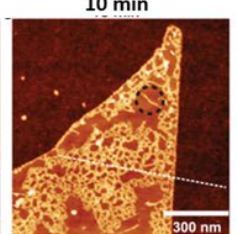

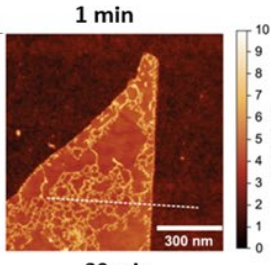

$30 \mathrm{~min}$

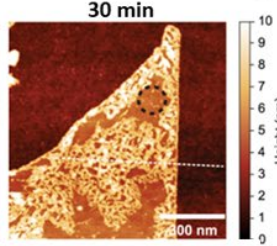

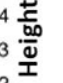
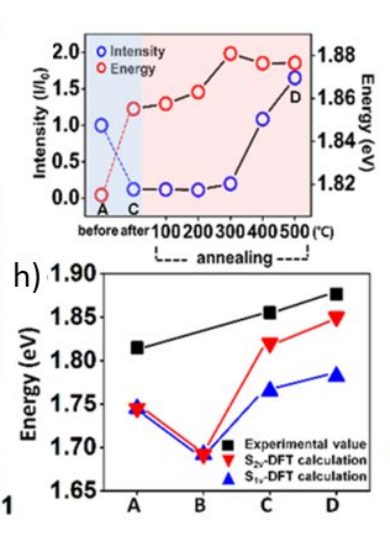

Chemical Structure Change (Input)

i)

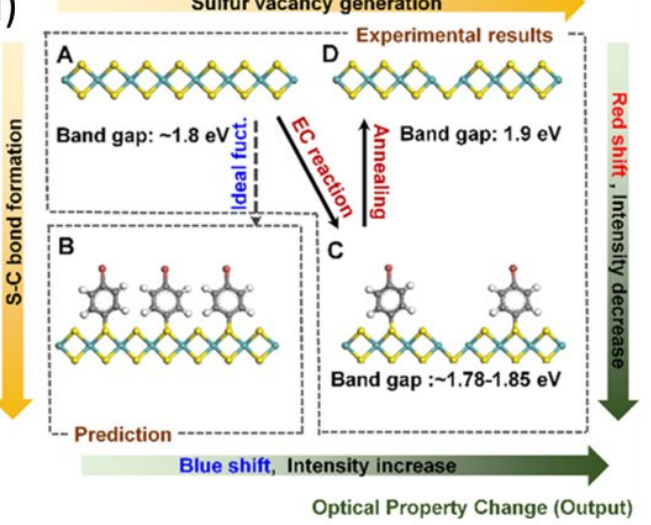

Figure 11. a) Scheme of the functionalization of $\mathrm{MoS}_{2}$ with NBD; b) optical microscope images of mechanically exfoliated flakes and corresponding AFM image of the marked area; c) PL maps before (left) and after (right) the functionalization process; d) AFM images before (left) and after (right) the functionalization of the surface. Reproduced with permission from reference 78. Copyright 2018 American Chemical Society. e) AFM images showing the evolution of the coverage on the surface with time for a $1 \mathrm{mM}$ NBD solution; f) Adsorption isotherms for the spontaneous adsorption of NBD on $\mathrm{MoS}_{2}$. Reproduced with permission from reference 79. Copyright 2019 American Chemical Society. g) PL spectra for functionalized $2 \mathrm{H}-\mathrm{MoS}_{2}$ after annealing at different temperatures and plot of the corresponding PL intensities and peak position; h) Theoretical and experimental band gap values for the different situations shown in scheme i); i) scheme of the mechanism showing the influence of the vacancies and the changes on 
the optical properties of the material. Reproduced with permission from reference 80. Copyright 2020 American Chemical Society.

As commented earlier, the functionalization using spontaneous decomposition of aryl diazonium ions can be achieved. Nevertheless, although the efficiency of this process is high, it induces the growth of a polymer on the surface in an uncontrolled fashion. For conductive surfaces, the activation and control of the decomposition process can be achieved by means of electrochemical techniques. However, the semiconducting behaviour of $2 \mathrm{H}-\mathrm{MoS}_{2}$ makes this approach extremely difficult due to the highly-impeded electron transfer through the material for the molecules to react. Recently, these difficulties could be overcome, allowing the electrochemical functionalization of $\mathrm{MoS}_{2}$ surfaces with 4-bromobenzene diazonium tetrafluoroborate (4-BBDT) by means of cyclic voltammetry. ${ }^{104}$ The deposition of a monolayer of $2 \mathrm{H}-\mathrm{MoS}_{2}$ on platinum allows the electron transfer to occur through an electron tunnelling mechanism, promoting the reduction of the diazonium ions at the interface. Interestingly, after the formation of the C$\mathrm{S}$ bond on the surface, the electronic structure of the material can be tuned. PL measurements show that the electrochemical process promotes the functionalization of the material but creates defects (sulphur vacancies) on the surface as well. The annealing of the functionalized samples up to $400^{\circ} \mathrm{C}$ shows the recovery of the $\mathrm{PL}$ intensity. A possible mechanism to explain these changes in the optoelectronic properties is shown in Figure $10 \mathrm{~g}$-i.

The substituent in the precursor for the covalent modification has been found to influence the performance of the $1 \mathrm{~T}-\mathrm{MOS}_{2}$ phase as electrocatalyst for the hydrogen evolution reaction (HER). ${ }^{105}$ HER activity and stability of the functionalized surfaces were analyzed for $1 \mathrm{~T}-\mathrm{MoS}_{2}$ carrying different functionalities (Et $2 \mathrm{~N}$-, $\mathrm{MeO}-, \mathrm{Br}_{-}, \mathrm{Cl}_{2-}$ and $\mathrm{NO}_{2-}$ ) with different electron donating / withdrawing properties. The electrocatalytic activity, evaluated in terms of Tafel slope and the overpotential required to reach a current density of 10 $\mathrm{mA} / \mathrm{cm}^{2}$, strongly depends on the functional group on the surface. The best performance among the functionalized substrates was found for the Et 2 NPh-MoS 2 (Tafel slope: $75 \pm 3 \mathrm{mV} / \mathrm{dec}$ ) case, showing a small decrease when compared to the bulk 1T-MoS 2 phase. Electron-withdrawing groups lead to a decrease in the electrocatalytic activity with a consequent increase of the Tafel slope (Tafel slope for $\mathrm{Cl}_{2} \mathrm{Ph}-\mathrm{MoS}_{2}=213$ $\pm 33 \mathrm{mV} / \mathrm{dec}$ ) and the overpotential. When the stability of the material under HER conditions of the material was evaluated, the stability of the $1 \mathrm{~T}-\mathrm{MoS}_{2}$ phase in the $\mathrm{Et}_{2} \mathrm{NPh}$ modified materials was confirmed.

These materials can be post-functionalized to form complex organic-inorganic hybrid interfaces. One of the easiest and more versatile ways is the use of click chemistry on the already grafted molecules on the surface. Aryl diazonium chemistry has been exploited for the modification of $\mathrm{MoS}_{2}$ nanosheets with phenylazido moieties. ${ }^{106}$ In a second step, through a copper-mediated azide-alkyne cycloaddition, single terminal acetylenes and defined binary acetylene mixtures are integrated to the surface. This strategy has been proved to be efficient to anchor an extensive number of functional groups on the surface and even more than one functional group when alkyne mixtures are used. A battery of techniques has been used for the characterization of the modified materials (SEM microscopy, Raman, XPS, UV-vis, etc.). Interestingly, fluorescent labeling of the material has been achieved with this method which allows the tracking of the 
functionalization by fluorescence lifetime imaging (FLIM). The fluorescence images show a good distribution of the dye through the sample, confirming the efficiency of the grafting of the diazonium ions and the successful coupling of the alkyne cycloaddition. These modifications have been confirmed additionally by using XPS measurements where the specific signals for the functional groups introduced can be found. The post-synthetic functionalization of aryl diazonium-based platforms represent a simple and versatile method for the fabrication of multifunctional 2D inorganic materials.

Aryl diazonium salts have been employed for the functionalization of other TMDCs and pnictogen chalcogenides (PC). ${ }^{98}$ The possibility of expanding the use of diazonium ions to a broad number of different materials has been proved. In all the cases the functionalized materials have been characterized by spectroscopic and microscopic methods showing the efficiency of the molecular modification. FTIR and XPS confirms the presence of the molecule on the surface and the covalent attachment. Raman spectroscopy experiments have shown that the crystal structure of the material remains unaltered after the functionalization. It is remarkable that this chemistry works in both water and solution-based materials representing a valuable tool for the functionalization of different 2D materials. In fact, other popular 2D layered materials such as BP and h-BN have been modified using this approach. In the case of BP this covalent functionalization allows the passivation of the material. ${ }^{99}$ This passivation is of great importance due to the well-known chemical instability of BP. It avoids the surface degradation under ambient conditions. This can be analysed by observing the morphological changes on the surface with time. It has been proved that after the modification, confirmed by the formation of P-C bonds in XPS, there is a clear difference in the evolution of the morphology of the surface with time, as confirmed by AFM images. When the surface is not modified, after 10 days of exposure of the samples to ambient conditions, the appearance of large morphological features appear on the surface. In contrast, when NBD groups are anchored on the surface, the sample morphology does not show differences when compared to the images for fresh samples. The surface roughness through AFM experiments for the non-modified and modified flakes confirm the observations. For the NBD modified samples, the invariance of the morphology is maintained for at least 25 days. The functionalization has been proved to produce significant changes in the electronic properties of the exfoliated BP. Especially at low degrees of functionalization, these changes result in an enhanced semiconductor behaviour, with an improvement in the hole mobility and on/off current ratio in FETS devices.

\subsection{MALEIMIDES}

Maleimides are electrophilic compounds with a well-known high selectivity toward thiols. ${ }^{107}$ These chemical compounds can be conjugated to sulfhydryl groups, a reaction widely used for the covalent bonding of proteins. With this knowledge, it seems that these molecules are suitable for the functionalization of 2D materials containing sulphur in its structure. Recently, a mild chemistry based on the use of maleimides has been proposed for the covalent modification of $2 \mathrm{H}-\mathrm{MoS}_{2}$ and $\mathrm{WS}_{2}$. This reaction is based on the soft nucleophilicity of the sulphur to react with a soft electrophile such as the maleimide compounds via a Michael addition. ${ }^{108}$ The advantages of this method rely on the use of mild conditions, the 
possibility to functionalize pristine surfaces, the short times needed for the complete functionalization and its versatility. The surface coverage has been calculated using the S $2 p(33 \%)$ and the Mo $3 d(25 \%)$ regions in XPS. These values are in good agreement with those estimated using TGA measurements (21\%) and are similar to the ones obtained for other TMDCs such as WS2. As a consequence, with this protocol it is possible to obtain a highly efficient covalent attachment without the use of harsh conditions or the transformation of the $2 \mathrm{H}-\mathrm{MOS}_{2}$ phase to the highly-reactive $1 \mathrm{~T}-\mathrm{MoS}_{2}$ one. In fact, after the nanosheet functionalization HRTEM measurements reveal that the $2 \mathrm{H}-\mathrm{MoS}_{2}$ phase remains the dominant phase, proving this method as a suitable tool for the modification of the basal plane of TMDC.

After this first proof of concept, Pérez and coworkers evaluated the performance of different precursors based on this maleimide covalent chemistry. ${ }^{109}$ They showed that the reaction can undergo two different pathways if a base such as $\mathrm{Et}_{3} \mathrm{~N}$ is used giving rise to polymeric growth (in presence of base) or limited growth to the monolayer (in absence of base). (Figure 12) Additionally, the versatility of the reaction was tested by using different precursors demonstrating that the maleimide chemistry is suitable to introduce different functional groups on the basal plane of $2 \mathrm{H}-\mathrm{TMDC}$. Due to the commercially available reagents to produce protein-polymer conjugates based on maleimides, this chemistry is an interesting and promising tool for their incorporation into these TMDC materials.

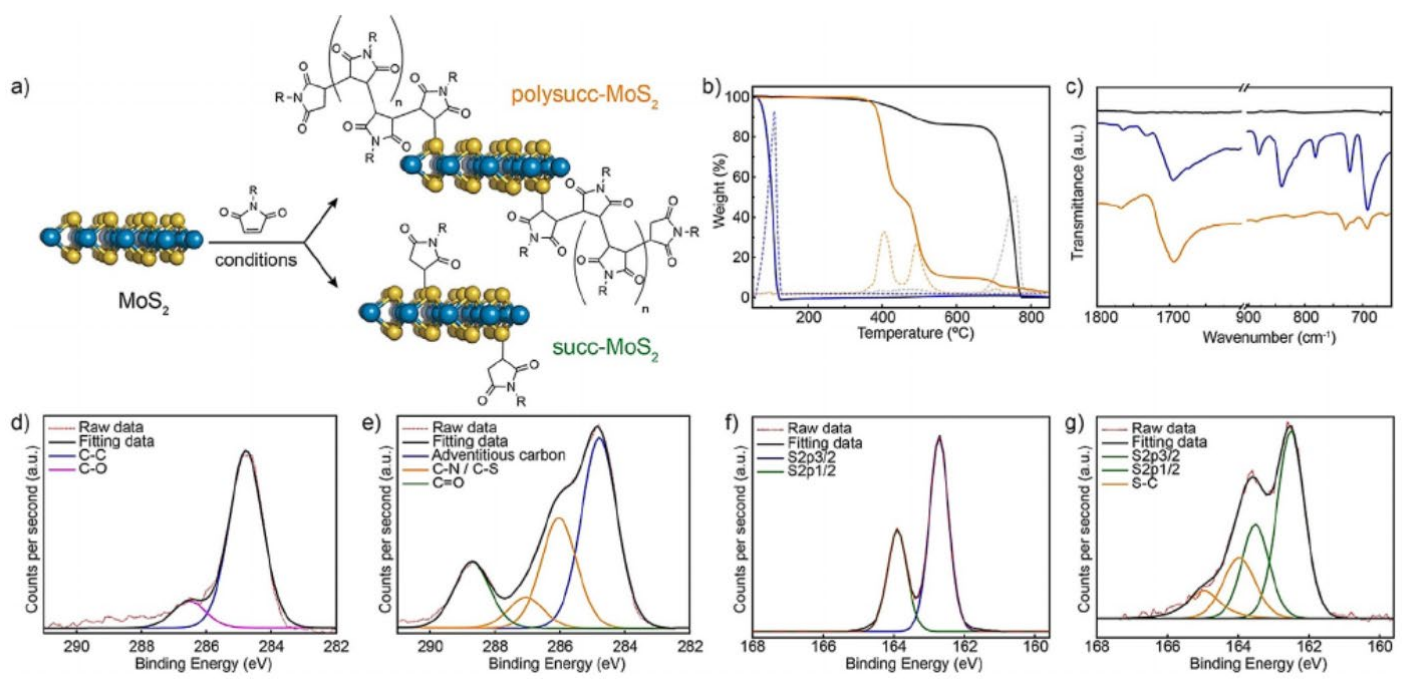

Figure 12. a) Scheme of the functionalization of $\mathrm{MoS}_{2}$ with maleimides through two different routes. b) TGA of exfoliated flakes (black) and functionalized ones (Bn-poly-succ-MoS ${ }_{2}=$ orange; Bn-mal =blue); c) ATR-FTIR of the same materials. (d-e) XPS spectra for C core levels for bare and functionalized flakes, respectively and (f-g) XPS spectra for the $\mathrm{S}$ core levels of the same samples. Reproduced with permission from reference 85. Copyright 2020 Wiley VCH.

\section{CONCLUSIONS AND FUTURE PERSPECTIVES}

Chemical functionalization of 2D materials is a field with plenty of possibilities and with a rapid growth in the last years. When compared to the functionalization of graphene surfaces, the modification of these materials using molecules has been much less explored. Taking into account the availability of molecular 
building blocks that can be attached to TMDCs, there is plenty of room for the fundamental understanding of the chemical nature of these new interfaces and the changes in the properties of the functionalized materials. The future challenges in terms of molecular functionalization of 2D materials lie in the control of the structure and properties at the molecular level. The possibility of post-functionalization of the surfaces with covalently incorporated enzymes, antibodies or DNA can lead to the production of bio-functional surfaces with relevance in biomedicine. The use of $\mathrm{MoS}_{2}$ in this field is especially promising due to its biocompatibility. ${ }^{110,111}$ Additionally, control of the nature and the level of doping or band gap through molecular modification can bring new functional materials in fields such as nanoelectronics or electrocatalysis. From the fundamental point of view, new insights in the functionalization of the 1T-MoS2 phase are expected in the next years. To date most studies have been focused on the thermodynamically stable $2 \mathrm{H}-\mathrm{MoS}_{2}$ phase. Nevertheless, regarding other prototypes of $\mathrm{MoS}_{2}$, the $1 \mathrm{~T}-\mathrm{MoS}_{2}$ has interesting properties as electrocatalyst and the $3 R$ has exciting optical properties. Finally, in terms of covalent functionalization there is a need of new characterization techniques which can be used as standard protocols for the quantification of the degree of functionalization, in the same way Raman spectroscopy is employed for the characterization of modified graphene. It is therefore expected that research on the functionalization of 2D materials will keep growing and be extended to new materials, molecular building blocks and the use of novel techniques.

\section{REFERENCES}

(1) K. S Novoselov; A. K. Geim; S. v. Morozov; D. Jiang; Y. Zhang; S. v. Dubonos; I. v. Grigorieva; A. A. Firsov. Science 306 (5696) (2004), 666-669

(2) K. Khan, A. K. Tareen, M. Aslam, R. Wang, Y. Zhang, A. Mahmood, Z. Ouyang, H. Zhang and Z. Guo. J. Mater. Chem. C 8 (2) (2020) 387-440.

(3) S. Manzeli, D. Ovchinnikov, D. Pasquier, O. V. Yazyev and A. Kis. Nat. Rev. Mater. 8 (2017) 1-15.

(4) J. Wang, F. Ma and M. Sun. RSC Adv. 27 (2017) 16801-16822.

(5) F. Xia, H. Wang, J. C. M. Hwang, A. H. C. Neto and L. Yang. Nat. Rev. Phys. 1 (2019) 306-317.

(6) A. Acun, L. Zhang, P. Bampoulis, M. Farmanbar, A. van Houselt, A. N. Rudenko, M. Lingenfelder, G. Brocks, B. Poelsema, M. I. Katsnelson and H. J. W. Zandvliet. J. Phys. Condens. Matter. 27 (2015) 443002.

(7) Q. Tang and D. E. Jiang. Chem. Mater. 27(10) (2015) 3743-3748.

(8) Z. Song; T. Schultz; Z. Ding; B. Lei; C. Han; P. Amsalem; T. Lin; D. Chi; S. L. Wong; Y. J. Zheng; M. Y. Li; L. J. Li; W. Chen; N. Koch; Y. L. Huang; A. T. S. Wee. ACS Nano 11 (9) (2017) 9128-9135

(9) L. O. Jones, M. A. Mosquera, M. A. Ratner and G. C. Schatz. ACS Appl. Mater. Interfaces. 12 (4) (2020) 4607-4615.

(10) D. Voiry, A. Goswami, R. Kappera, C. D. C. C. E. Silva, D. Kaplan, T. Fujita, M. Chen, T. Asefa and M. Chhowalla. Nat. Chem. 7 (1) (2015) 45-49.

(11) X. Ding and J. Yi. Synthesis and Applications of $2 d$ Transition Metal Dichalcogenides, Functional Materials and Electronics, (Apple Academic Press, 2018) pp 1-33.

(12) M. J. Park, S. Gravelsins, J. Son, A. M. van der Zande and A. A. Dhirani. ACS Nano 13 (6) (2019) 64696476.

(13) I. Song, C. Park and H. C. Choi. RSC Adv. 5 (2015) 7495-7514. 
(14) S. Bertolazzi, M. Gobbi, Y. Zhao, C. Backes and P. Samorì. Chem. Soc. Rev. 47(17) (2018) 6845-6888.

(15) J. Ristein. Science. 313 (2006) 1057-1058.

(16) S. Tongay, J. Zhou, C. Ataca, J. Liu, J. S. Kang, T. S. Matthews, L. You, J. Li, J. C. Grossman and J. Wu, J. Nano Letters 13(6) (2013) 2831-2836.

(17) D. J. Late, B. Liu, H. S. S. R. Matte, V. P. Dravid and C. N. R. Rao. ACS Nano 6(6) (2012) 5635-5641.

(18) J. O. Varghese, P. Agbo, A. M. Sutherland, V. W. Brar, G. R. Rossman, H. B. Gray and J. R. Heath. Adv. Mater 27(17) (2015) 2734-2740.

(19) Y. Wang, A. Slassi, M. A. Stoeckel, S. Bertolazzi, J. Cornil, D. Beljonne and P. Samorì, P. J. Phys. Chem. Lett. 10(3) (2019) 540-547.

(20) X. Li, R. Sun, H. Guo, B. Su, D. Li, X. Yan, Z. Liu and J. Tian. Adv. Electron. Mater. 6(3) (2020) 1901230.

(21) J. Zhao, N. Li, H. Yu, Z. Wei, M. Liao, P. Chen, S. Wang, D. Shi, Q. Sun and G. Zhang. Adv. Mater 29(34) (2017) 1702076.

(22) S. Y. Lee, U. J. Kim, J. Chung, H. Nam, H. Y. Jeong, G. H. Han, H. Kim, H. M. Oh, H. Lee, H. Kim, Y. G. Roh, J. Kim, S. W. Hwang, Y. Park and Y. H. Lee. ACS Nano 10(6) (2016) 6100-6107.

(23) A. K. Singh, S. Andleeb, J. Singh, H. T. Dung, Y. Seo and J. Eom. Adv. Funct. Mater 24(45) (2014) 71257132.

(24) S. Zhao; J. Xue; W. Kang. Chem Phys Lett 595-592 (2014) 35-42

(25) D. P. Goronzy, M. Ebrahimi, F. Rosei, Arramel; Y. Fang, S. de Feyter, S. L. Tait, C. Wang, P. H. Beton, A. T. S. Wee, P. S. Weiss and D. F. Perepichka. ACS Nano 12(8) (2018) 7445-7481.

(26) Y. Li, C. Y. Xu, P. Hu and L. Zhen. ACS Nano 7(9) (2013) 7795-7804.

(27) D. H. Kang, M. S. Kim, J. Shim, J. Jeon, H. Y. Park, W. S. Jung, H. Y. Yu, C. H. Pang, S. Lee and J. H. Park. Adv. Funct. Mater 25(27) (2015) 4219-4227.

(28) D. H. Kang, J. Shim, S. K. Jang, J. Jeon, M. H. Jeon, G. Y. Yeom, W. S. Jung, Y. H. Jang, S. Lee and J. H. Park. ACS Nano 9(2) (2015) 1099-1107.

(29) J. Li, J. Wierzbowski, Ö. Ceylan, J. Klein, F. Nisic, T. le Anh, F. Meggendorfer, C. A. Palma, C. Dragonetti, J. v. Barth, J. J. Finley and E. Margapoti. Appl. Phys. Lett. 105(24) (2014) 241116.

(30) E. Margapoti, J. Li, Ö. Ceylan, M. Seifert, F. Nisic, T. le Anh, F. Meggendorfer, C. Dragonetti, C. A. Palma, J. v. Barth and J. J. Finley. Adv. Mater. 27(8) (2015) 1426-1431.

(31) D. Wöhrle, L. Kreienhoop, G. Schnurpfeil, J. Elbe, B. Tennigkeit, S. Hiller and D. Schlettwein. J. Mater. Chem. 5(11) (1995) 1819-1829.

(32) N. Karousis, J. Ortiz, K. Ohkubo, T. Hasobe, S. Fukuzumi, A. Sastre-Santos and N. Tagmatarchis. J. Phys. Chem. C 116(38) (2012) 20564-20573.

(33) Y. Kaneda, M. E. Stawasz, D. L. Sampson and B. A. Parkinson. Langmuir 17(20) (2001) 6185-6195.

(34) C. Wirtz, T. Hallam, C. P. Cullen, N. C. Berner, M. O’Brien, M. Marcia, A. Hirsch and G. S. Duesberg. Chem. Comm. 51(92) (2015) 16553-16556.

(35) A. J. Molina-Mendoza, L. Vaquero-Garzon, S. Leret, L. de Juan-Fernández, E. M. Pérez and A. CastellanosGomez. Chem. Comm. 52(100) (2016) 14365-14368.

(36) H. Zhang, J. Choi, A. Ramani, D. Voiry, S. N. Natoli, M. Chhowalla, D. R. McMillin and J. H. Choi. ChemPhysChem 17(18) (2016) 2854-2862.

(37) J. Choi, H. Zhang and J. H. Choi. ACS Nano 10(1) (2016) 1671-1680.

(38) Y. Wang, S. M. Gali, A. Slassi, D. Beljonne and P. Samorì. Adv. Funct. Mat. 30 (2020) 2002846. 
E. P. Nguyen, B. J. Carey, C. J. Harrison, P. Atkin, K. J. Berean, E. della Gaspera, J. Z. Ou, R. B. Kaner, K. Kalantar-Zadeh and T. Daeneke. Nanoscale 8(36) (2016) 16276-16283.

(40) S. Mouri, Y. Miyauchi and K. Matsuda. Nano Lett. 13(12) (2013) 5944-5948.

(41) D. Kiriya, M. Tosun, P. Zhao, J. S. Kang and A. Javey. J.Am. Chem. Soc. 136(22) (2014) 7853-7856.

(42) Y. Huang, W. Zheng, Y. Qiu and P. Hu. ACS Appl. Mat. Inter. 8(35) (2016) 23362-23370.

(43) K. Heo, S. H. Jo, J. Shim, D. H. Kang, J. H. Kim and J. H. Park. ACS Appl. Mat. Inter. 10(38) (2018) 3276532772.

(44) C. J. Lockhart De La Rosa, R. Phillipson, J. Teyssandier, J. Adisoejoso, Y. Balaji, C. Huyghebaert, I. Radu, M. Heyns, S. de Feyter and S. de Gendt. App. Phys. Lett. 109(25) (2016) 253112.

(45) R. C. Selhorst, E. Puodziukynaite, J. A. Dewey, P. Wang, M. D. Barnes, A. Ramasubramaniam and T. Emrick. Chem. Sci. 7(7) (2016) 4698-4705.

(46) H. Y. Park, S. R. Dugasani, D. H. Kang, J. Jeon, S. K. Jang, S. Lee, Y. Roh, S. H. Park and J. H. Park. ACS Nano 8(11) (2014) 11603-11613.

(47) K. H. Han, J. Y. Kim, S. G. Jo, C. Seo, J. Kim; J. Joo. Nanotechnology 28(43) (2017) 435501.

(48) Y. J. Zheng; Y. L. Huang; Y. Chen; W. Zhao; G. Eda; C. D. Spataru; W. Zhang; Y. H. Chang; L. J. Li; D. Chi; S. Y. Quek; A. T. S. Wee. ACS Nano 10(2) (2016) 2476-2484.

(49) X. Lin; J. C. Lu; Y. Shao; Y. Y. Zhang; X. Wu; J. B. Pan; L. Gao; S. Y. Zhu; K. Qian; Y. F. Zhang; D. L. Bao; L. F. Li; Y. Q. Wang; Z. L. Liu; J. T. Sun; T. Lei; C. Liu; J. O. Wang; K. Ibrahim; D. N. Leonard; W. Zhou; H. M. Guo; Y. L. Wang; S. X. Du; S. T. Pantelides; H. J. Gao. Nat. Mater 16(7) (2017) 717-721.

(50) D. Jariwala; S. L. Howell; K. S. Chen; J. Kang; V. K. Sangwan; S. A. Filippone; R. Turrisi; T. J. Marks; L. J. Lauhon; M. C. Hersam. Nano Lett 16(1) (2016) 497-503

(51) Y. Zhang; L. Xu; W. R. Walker; C. M. Tittle; C. J. Backhouse; M. A. Pope. J Mat Chem C 5(43) (2017) 1127511287.

(52) D. Li; W. Zhou; Q. Zhou; G. Ye; T. Wang; J. Wu; Y. Chang; J. Xu. Nanotechnology 28(39) (2017) 395401.

(53) J. Wu; H. Zeng; X. Li; X. Xiang; Y. Liao; Z. Xue; Y. Ye; X. Xie. Adv Energy Mater 8(35) (2018) 2018, 8 (35) 1802430

(54) P. Joo, K. Jo, G. Ahn, D. Voiry, H. Y. Jeong, S. Ryu, M. Chhowalla and B. S. Kim. Nano Lett. 14(11) (2014) 6456-6462.

(55) S. Zhang, W. Wang, L. Guan, X. Niu, J. Yao, X. Yan, B. Xing, Y. Yu, J. Sha and Y. Wang. J. Lumin. 213 (2019) 388-394.

(56) W. Bao, X. Cai, D. Kim, K. Sridhara and M. S. Fuhrer. App. Phys. Lett. 102(4) (2013) 042104.

(57) B. Liu, W. Zhao, Z. Ding, I. Verzhbitskiy, L. Li, J. Lu, J. Chen, G. Eda and K. P. Loh. Adv. Mater. 28(30) (2016) 6457-6464.

(58) H. Kim, D. H. Lien, M. Amani, J. W. Ager and A. Javey. ACS Nano 11(5) (2017) 5179-5185.

(59) J. Lu; M. Chen; L. Dong; L. Cai; M. Zhao; Q. Wang; J. Li. Colloids Surf. B 194 (2020) 111162.

(60) J. Liu; Z. Zeng; X. Cao; G. Lu; L. H. Wang; Q. L. Fan; W. Huang; H. Zhang. Small 8(22) (2012) 3517-3522.

(61) X. Zhang; Z. Lai; Z. Liu; C. Tan; Y. Huang; B. Li; M. Zhao; L. Xie; W. Huang; H. Zhang. Angew. Chem. Int. Ed. 54(18) (2015) 5425-5428.

(62) Y. Yao; L. Tolentino; Z. Yang; X. Song; W. Zhang; Y. Chen; C. P. Wong. Adv. Funct. Mater. 23(28) (2013) 3577-3583.

(63) H. Dong; D. Chen; K. Wang; R. Zhang. Nanoscale Res. Lett. 11(1) (2016) 409. 
(64) G. Guan; S. Zhang; S. Liu; Y. Cai; M. Low; C. P. Teng; I. Y. Phang; Y. Cheng; K. L. Duei; B. M. Srinivasan; Y. Zheng; Y. W. Zhang; M. Y. Han. J Am Chem Soc 137(19) (2015) 6152-6155.

(65) Y. Yong; L. Zhou; Z. Gu; L. Yan; G. Tian; X. Zheng; X. Liu; X. Zhang; J. Shi; W. Cong; W. Yin; Y. Zhao. Nanoscale 6(17) (2014) 10394-10403.

(66) Y. Ge; J. Wang; Z. Shi; J. Yin. J. Mat Chem 22(34) (2012) 17619-17624.

(67) M. C. Demirel; M. Vural; M. Terrones. Adv Funct Mat 28(27) (2018) 1704990.

(68) H. Chen; T. Liu; Z. Su; L. Shang; G. Wei. Nanoscale Horiz. 3 (2018) 74-89

(69) J. Lee; P. Dak; Y. Lee; H. Park; W. Choi; M. A. Alam; S. Kim. Sci. Rep 4(1) 2014, 1-7.

(70) J. Ma, K. Y. Choi, S. H. Kim, H. Lee and G. Yoo. Appl. Phys. Lett. 113(1) (2018) 013102

(71) K. Zhou, J. Liu, P. Wen, Y. Hu and Z. Gui. Appl. Surf. Sci. 316(1) (2014) 237-244

(72) J. Biscarat; M. Bechelany; C. Pochat-Bohatier; P. Miele. Nanoscale 7(2) (2015) 613-618.

(73) Y. Lin; T. v. Williams; T. B. Xu; W. Cao; H. E. Elsayed-Ali; J. W. Connell. J Phys Chem C 115(6) (2011) 26792685.

(74) A. Ramasubramaniam; R. Selhorst; H. Alon; M. D. Barnes; T. Emrick; D. Naveh. J Mat Chem C 5(43) 2017 11158-11164.

(75) W. T. Cao; F. F. Chen; Y. J. Zhu; Y. G. Zhang; Y. Y. Jiang; M. G. Ma; F. Chen. ACS Nano 12(5) (2018) 45834593.

(76) A. Criado, M. Melchionna, S. Marchesan and M. Prato. The Covalent Functionalization of Graphene on Substrates. Angew. Chem. Int. Ed. 54(37) (2015) 10734-10750.

(77) A. Hirsch and F. Hauke. Angew. Chem. Int. Ed. 57(16) (2018) 4338-4354.

(78) S. S. Chou, M. De, J. Kim, S. Byun, C. Dykstra, J. Yu, J. Huang and V. P. Dravid. J. Am. Chem. Soc. 135(12) (2013) 4584-4587.

(79) Y. T. Liu, Q. P. Feng, X. M. Xie and X. Y. Ye. Carbon 49 (2011) 3371-3375.

(80) (Y. T. Liu, Z. Tan, X. M. Xie, Z. F. Wang and X. Y. Ye. Chem. Asian J. 8(4) (2013) 817-823.

(81) C. Backes, N. C. Berner, X. Chen, P. Lafargue, P. LaPlace, M. Freeley, G. S. Duesberg, J. N. Coleman, A. R. McDonald. Angew. Chem. Int. Ed. 54(9) (2015) 2638-2642.

(82) C. Vericat, M. E. Vela, G. Benitez, P. Carro and R. C. Salvarezza. Chem. Soc. Rev. 39(5) (2010) 1805-1834.

(83) L. Soldi, R. J. Cullen, D. R. Jayasundara, E. M. Scanlan, S. Giordani and P. E. Colavita. J. Phys. Chem. C 115(20) (2011) 10196-10204.

(84) E. P. Nguyen, B. J. Carey, J. Z. Ou, J. van Embden, E. della Gaspera, A. F. Chrimes, M. J. Spencer, S. Zhuiykov, K. Kalantar-Zadeh and T. Daeneke. Adv. Mater. 27(40) (2015) 6225-6229.

(85) X. Chen, N. C. Berner, C. Backes, G. S. Duesberg and A. R. McDonald. Angew. Chem. Int. Ed. 55(19) (2016) 5803-5808.

(86) A. Förster, S. Gemming, G. Seifert and D. Tománek. ACS Nano 11(10) (2017) 9989-9996.

(87) X. Chen, P. Denninger, T. Stimpel-Lindner, E. Spiecker, G. S. Duesberg, C. Backes, K. C. Knirsch and A. Hirsch. Chem. Eur. J. 26(29) 2020, 6535-6544.

(88) Q. Ding, K. J. Czech, Y. Zhao, J. Zhai, R. J. Hamers, J. C. Wright and S. Jin. ACS Appl. Mat. Inter. 9(14) (2017) 12734-12742.

(89) R. Canton-Vitoria, Y. Sayed-Ahmad-Baraza, M. Pelaez-Fernandez, R. Arenal, C. Bittencourt, C. P. Ewels and N. Tagmatarchis. NPJ 2D Mater. Appl. 1(1) (2017) 13.

(90) M. A. Gerkman, J.K. Lee, X. Li, Q. Zhang, M. Windley, M. v. Fonseca, Y. Lu, J. H. Warner and G. D. Han. ACS Nano 14(1) (2020) 153-165. 
(91) I. K. Sideri, R. Arenal and N. Tagmatarchis. ACS Mat. Lett. 2(7) (2020) 832-837.

(92) R. Canton-Vitoria, C. Stangel and N. Tagmatarchis. ACS Appl. Mat. Inter. 10(28) (2018) 23476-23480.

(93) M. Blanco, M. Lunardon, M. Bortoli, D. Mosconi, L. Girardi, L. Orian, S. Agnoli and G. Granozzi. J. Mater. Chem. A 8 (2020) 11019-11030.

(94) P. Vishnoi, A. Sampath, U. v. Waghmare and C. N. R. Rao. Chem. Eur. J. 23(4) (2017) 886-895.

(95) E. X. Yan, M. Cabán-Acevedo, K. M. Papadantonakis, B. S. Brunschwig and N. S. Lewis. ACS Mater. Lett. 2(2) (2020) 133-139.

(96) G. Bottari, M. Ángeles Herranz, L. Wibmer, M. Volland, L. Rodríguez-Pérez, D. M. Guldi, A. Hirsch, N. Martín, F. D'Souza and T. Torres. Chem. Soc. Rev. 46 (2017) 4464-4500.

(97) J. M. Englert, C. Dotzer, G. Yang, M. Schmid, C. Papp, J. M. Gottfried, H. P. Steinrück, E. Spiecker, F. Hauke and A. Hirsch. Nat. Chem. 3(4) (2011) 279-286.

(98) D. O. Li, M. S. Gilliam, X. S. Chu, A. Yousaf, Y. Guo, A. A. Green Bc and Q. H. Wang. Mol. Syst. Des. Eng. 4 (2019) 962.

(99) C. R. Ryder, J. D. Wood, S. A. Wells, Y. Yang, D. Jariwala, T. J. Marks, G. C. Schatz and M. C. Hersam. Nat. Chem. 8(6) (2016) 597-602.

(100) Z. Wang, Q. Li, Z. Chen, H. Li, S. Zheng and X. Tang. Polym. Compos. 40(6) (2019) 2346-2356.

(101) K. C. Knirsch, N. C. Berner, H. C. Nerl, C. S. Cucinotta, Z. Gholamvand, N. McEvoy, Z. Wang, I. Abramovic, P. Vecera, M. Halik, S. Sanvito, G. S. Duesberg, V. Nicolosi, F. Hauke, A. Hirsch, J. N. Coleman and C. Backes. 9(6) (2015) ACS Nano 6018-6030.

(102) X. S. Chu, A. Yousaf, D. O. Li, A. A. Tang, A. Debnath, D. Ma, A. A. Green, E. J. G. Santos and Q. H. Wang. Chem. Mat. 30(6) (2018) 2112-2128.

(103) D. O. Li, X. S. Chu and Q. H. Wang. Langmuir 35(17) (2019) 5693-5701.

(104) Y. Park, S. Shin, Y. An, J. G. Ahn, G. Shin, C. Ahn, J. Bang, J. Baik, Y. Kim, J. Jung and H. Lim. ACS Appl. Mater. Inter. 12(36) (2020) 40870-40878.

(105) E. E. Benson, H. Zhang, S. A. Schuman, S. U. Nanayakkara, N. D. Bronstein, S. Ferrere, J. L. Blackburn and E. M. Miller. J. Am. Chem. Soc. 140(1) (2018) 441-450.

(106) G. Tuci, D. Mosconi, A. Rossin, L. Luconi, S. Agnoli, M. Righetto, C. Pham-Huu, H. Ba, S. Cicchi, G. Granozzi and G. Giambastiani. Chem. Mat. 30(22) (2018) 8257-8269.

(107) B. H. Northrop, S. H. Frayne and U. Choudhary. Polym. Chem. 6(18) (2015) 3415-3430.

(108) M. Vera-Hidalgo, E. Giovanelli, C. Navío and E. M. Pérez. J. Am. Chem. Soc. 141(9) (2019) 3767-3771.

(109) R. Quirós-Ovies, M. Vázquez Sulleiro, M. Vera-Hidalgo, J. Prieto, I. J. Gómez, V. Sebastián, J. Santamaría and E. M. Pérez. Chem. Eur. J. 26(29) (2020) 6629-6634

(110) C. Martí; K. Kostarelos; M. Prato; A. Bianco. Chem. Commun 55 (2019) 5540--5546.

(111) J. H. Appel; D. O. Li; J. D. Podlevsky; A. Debnath; A. A. Green; Q. H. Wang; J. Chae. ACS Biomater. Sci. Eng. 2(3) (2016) 361-367. 